\title{
Similarity of the streamwise velocity component in very-rough-wall channel flow
}

\author{
DAVID M. BIRCH† AND JONATHAN F. MORRISON \\ Department of Aeronautics, Imperial College, London, SW7 2AZ, UK
}

(Received 21 April 2010; revised 1 September 2010; accepted 1 September 2010;

first published online 3 December 2010)

The streamwise velocity component is studied in fully developed turbulent channel flow for two very rough surfaces and a smooth surface at comparable Reynolds numbers. One rough surface comprises sparse and isotropic grit with a highly nonGaussian distribution. The other is a uniform mesh consisting of twisted rectangular elements which form a diamond pattern. The mean roughness heights ( \pm the standard deviation) are, respectively, about $76( \pm 42)$ and $145( \pm 150)$ wall units. The flow is shown to be two-dimensional and fully developed up to the fourth-order moment of velocity. The mean velocity profile over the grit surface exhibits self-similarity (in the form of a logarithmic law) within the limited range of $0.04 \leqslant y / h \leqslant 0.06$, but the profile over the mesh surface does not, even though the mean velocity deficit and higher moments (up to the fourth order) all exhibit outer scaling over both surfaces. The distinction between self-similarity and outer similarity is clarified and the importance of the former is explained. The wake strength is shown to increase slightly over the grit surface but decrease over the mesh surface. The latter result is contrary to recent measurements in rough-wall boundary layers. Single- and twopoint velocity correlations reveal the presence of large-scale streamwise structures with circulation in the plane orthogonal to the mean velocity. Spanwise correlation length scales are significantly larger than corresponding ones for both internal and external smooth-wall flows.

Key words: pipe flow boundary layer, turbulent boundary layers

\section{Introduction}

There has been considerable recent interest in the scaling of turbulent flows over rough walls, and specifically, the extent to which the details of the surface condition remain apparent in the turbulence statistics. The classical view, originally proposed by Hama (1954), Clauser (1956), Rotta (1962) and Townsend (1956, 1976), assumes that local inhomogeneities arising from the specific roughness geometry are confined to a 'roughness sublayer', analogous to the viscous sublayer found over smooth walls, the thickness of which is generally accepted to be about five roughness heights. Under these assumptions, any effects of the roughness on the turbulence away from the wall relative to the smooth-wall case must necessarily be attributable to the increase in the wall shear stress, $\tau_{w}$, alone. One can therefore expect that the outer-region flow (or

$\dagger$ Present address: Faculty of Engineering and Physical Sciences, University of Surrey, Guildford, GU2 7XH, UK. Email address for correspondence: d.birch@surrey.ac.uk 
at least the mean velocity deficit and second moment) will scale exclusively with the wall friction velocity $u_{\tau}=\sqrt{\tau_{w} / \rho}$, and $\delta$, the outer length scale.

However, if sufficiently large, the characteristic roughness height, $k$, replaces $v / u_{\tau}$ (where $v$ is the kinematic viscosity) as the dominant imposed surface length scale without necessarily precluding either self-similarity of the mean velocity profile or Townsend outer similarity provided that $k$ is small relative to $\delta$. When the roughness Reynolds number, $k^{+}=k u_{\tau} / v$, is sufficiently large (typically, $k^{+} \gtrsim 100$ ), the surface is described as 'fully rough', a condition under which the mean velocity profile may be described by a log law in which the additive roughness function asymptotes to a constant. The mean velocity profile will be self-similar if there is sufficient scale separation for asymptotic matching to yield a region in which independence from both imposed length scales, $k$ and $\delta$, can be demonstrated. Then, the usual log law may be expressed as

$$
U^{+}=\frac{U}{u_{\tau}}=\frac{1}{\kappa} \ln (y-d)^{+}+B-\Delta U^{+},
$$

where $y$ is the wall-normal distance relative to an arbitrary origin (here, taken to be at the bottom of the roughness elements), $d$ is a zero-plane displacement which represents the height at which momentum is extracted and is always smaller than $k$ (Jackson 1981), and $\kappa$ and $B$ are, respectively, the von Kármán constant and the smooth-wall additive constant. The Hama (1954) roughness function or velocity shift, $\Delta U^{+}$, is a function only of the size and geometry of the roughness. For surfaces which are fully rough, (1.1) may be recast in the form

$$
U^{+}=\frac{1}{\kappa} \ln \left(\frac{y-d}{y_{0}}\right),
$$

where $y_{0}$ is a roughness length which is typically of the order of $0.1 k$, but is geometryspecific. Equations (1.1) and (1.2) are entirely equivalent, as $\Delta U^{+}, B$ and $y_{0}$ are related by the expression

$$
\Delta U^{+}=\frac{1}{\kappa} \ln \left(y_{0}^{+}\right)+B .
$$

As Hama (1954) suggested, $\Delta U^{+}$is a direct measure of the surface forces and (1.3) shows how it is directly analogous to $y_{0}$ (Jackson 1981).

In outer variables, the log law may also be written as

$$
U_{c l}^{+}-U^{+}=-\frac{1}{\kappa} \ln \left(\frac{y-d}{\delta}\right)+B^{*},
$$

where $U_{c l}$ is the outer velocity scale (the mean centreline velocity), $\delta$ is used interchangeably with channel half-height, $h$, and $B^{*}$ is the smooth-wall additive constant for defect scaling and may be expressed in terms of $B$ and $\Delta U^{+}$as

$$
B^{*}=U_{c l}^{+}-\frac{1}{\kappa} \ln \left(h^{+}\right)-B+\Delta U^{+} .
$$

It is helpful to illustrate the meaning of self-similarity by indicating precisely what (1.1) and (1.4) imply: the simultaneous collapse and overlap of the inner and outer scaling requires that the mean flow is independent of both the inner and outer length scales. Therefore, the length scale in the log argument may be freely chosen but it is usually taken to be the dominant imposed length scale so that its influence on either of the additive constants is removed. Then, for a sufficient separation of scales, $B$ and 
$B^{*}$ tend to constants. In the context of rough-wall flows, the appropriate inner length scale is $k$, which can be replaced by $y_{0}$ if the surface is fully rough.

These arguments constitute a stronger condition for similarity than one which assumes $a$ priori that the effects of roughness are confined to the roughness sublayer, or that the effect of the large eddies near either a rough or a smooth surface is weak. In either circumstance, it is usually assumed that inner $\left(u_{\tau}, y\right)$ scaling suffices (assuming that $\delta \gg y \gg k \gg v / u_{\tau}$ ), and then the log law and the localequilibrium approximation may be derived by dimensional analysis alone. This weaker set of conditions therefore implies that the large scales have no influence near the wall, and that the roughness has no influence away from it; that is, the inner-outer interaction is so weak that it has no appreciable effect upon the fluid motion.

On the basis of a review of available data, Jiménez (2004) suggests that for relative roughness $k / \delta \lesssim 2.5 \%$, these weaker conditions are sufficient. Taking $k^{+} \gtrsim 100$ for a fully rough surface, then it is likely that self-similarity of the mean velocity profile is possible for $\delta^{+} \gtrsim 4000$. However, if $k / \delta<2.5 \%$, then self-similarity will occur at a lower Reynolds number than that at which the surface becomes fully rough. Alternatively, if $k / \delta>2.5 \%$ the surface will be fully rough before a log law can be expected. Assuming a log law to be established, both the Hama (1954) roughness function and the wake strength, $\Delta U_{\text {wake }}^{+}$, are defined by (1.1): taking the former to be a direct measure of the surface condition, it may then be supposed that the latter is invariant with surface roughness (Tani 1987).

It has long been accepted that inner scaling fails for higher-order statistics near the wall even for the case of smooth walls, though there are many examples demonstrating that the mean velocity is self-similar; see, for example, the reviews by Raupach, Antonia \& Rajagopalan (1991) and Jiménez (2004), as well as the more recent results of Shockling, Allen \& Smits (2006) and Schultz \& Flack (2007). Townsend (1961) explained this discrepancy by introducing the concept of 'inactive' motion (see also Bradshaw 1967; Morrison, Subramanian \& Bradshaw 1992), in which the 'top-down' effect (Hunt \& Morrison 2000) of the large-scale, non-shear-stress-bearing motion near the wall is assumed to be a low-frequency modulation of the shear-stressbearing, active motion by the low-wavenumber wall-parallel velocity components. Morrison (2007) has discussed these effects in terms of an inner-outer interaction and has shown that inactive motion is a first-order, linear approximation of an inherently nonlinear process.

Over a rough surface, the inner-outer interaction is likely to be dominated by a 'bottom-up' transport of momentum and energy away from the near-wall region. If the roughness is sufficiently large, this transport provides direct coupling between the inner and outer scales, and self-similarity of the mean velocity will not be possible. In the case of external flows, though, there is evidence that the boundary layer merely 'rides' above the rough surface: Flack, Schultz \& Connelly (2007) show that, as $k / \delta$ increases, the growth rate of the boundary layer increases as well. For internal flows, on the other hand, this is not possible. In fully developed channel flow, the momentum equation prescribes a streamwise static pressure gradient that cannot vary in the wall-normal direction, even though there is a wall-normal pressure gradient. The pressure drop across individual roughness elements is constrained to match the streamwise pressure gradient at the channel centreline, which is directly proportional to the surface shear stress. Townsend's outer similarity is therefore expected to be fairly robust for both internal and external flows, but perhaps for different reasons; in fact, Connelly, Schultz \& Flack (2006), Flack et al. (2007) and Castro (2007) have 
demonstrated collapse of the appropriately scaled mean velocities in a boundary layer for roughness heights $k / \delta$ as high as $20 \%$.

The extension of Townsend's outer similarity beyond low-order moments of the streamwise velocity component is a subject of continued debate. Krogstad, Antonia \& Browne (1992) compared the boundary layer developed over a smooth wall with that developed over a wall roughened by a wire mesh with $k / \delta \approx 2.1 \%$ : they found a significant increase in the wall-normal turbulence intensity, $\overline{v^{2}}$, through the outer layer, as well as an increase in the frequency and magnitude of sweep and ejection events. Krogstad \& Antonia (1994) later demonstrated that these observations could be attributed to a rotation of the large-scale structure towards the wall-normal axis relative to the smooth-wall case. These observations were corroborated by the particleimage velocimetry results of Keirsbulck et al. (2002) and the direct numerical channel simulations of Bhaganagar, Kim \& Coleman (2004), with $k / \delta=3.8$ and $5.4 \%$, respectively. Antonia \& Krogstad (2001) conducted measurements in the boundary layers developed over two walls with different roughnesses but with matching $\Delta U^{+}$, and found significant differences in the observed turbulence characteristics in the outer layer, though the surfaces had similar values of $k / \delta \approx 1.8$ and $2.1 \%$. However, the combined experimental and numerical study of Krogstad et al. (2005) found that, for a rough-wall channel with $k / \delta=3.4 \%$ and a surface topology similar to the transverse grooves of Bhaganagar et al. (2004), the influence of the roughness did not extend beyond approximately five roughness heights from the surface, and the outer layer was unaffected. The authors attributed the apparent discrepancy to fundamental differences between channel and boundary layer flows. This conclusion was also reached by Bakken et al. (2005) for similarly scaled roughness over a range of $R e_{\tau}$, as well as by Volino, Schultz \& Flack (2007), whose correlations showed no evidence of structural differences between the boundary layers over a smooth wall and a mesh-roughened wall geometrically similar to that of Krogstad \& Antonia (1994) with $k / \delta=1.4 \%$. Flack, Schultz \& Shapiro (2005) also showed that outer scaling was satisfied in a boundary layer for velocity statistics up to the third order for $k / \delta \leqslant 2.2 \%$.

For practical roughness, the scaling of roughness effects by $k^{+}$alone is too simple and the effects of such factors as roughness geometry, distribution or density must also be taken into account. While $k / \delta$ appears to provide a meaningful measure of the degree of inner-outer interaction, the results of $\mathrm{Wu} \&$ Christensen (2007) suggest that bulk-averaged geometric measures of the roughness (such as the mean surface height or the root-mean-square roughness height) only poorly characterize the influence of the roughness. By expressing the roughness size in terms of the equivalent sandgrain roughness, $k_{s}$ (which is associated with the total losses in fully developed turbulent pipe flow rather than any geometric parameter, Nikuradse 1933), Wu \& Christensen showed that the relative roughnesses, $k_{s} / \delta$, of Krogstad et al. (1992), Keirsbulck et al. (2002) and Bhaganagar et al. (2004) were 6.7, 13 and 13\%, respectively, and so that the application of the Townsend (1976) scaling assumptions may have been inappropriate in these cases. For the turbulent channel case of Krogstad et al. (2005), Wu \& Christensen noted that $k_{s} / \delta$ was as high as $25 \%$, rendering the lack of observed effects of the roughness upon the turbulent flow structures even more surprising, regardless of boundary conditions.

Clearly, the effect of large roughness upon turbulence structure is still not well understood, especially in the case of internal flows. The purpose of the present study is to address this issue by examining self-similarity of the mean velocity and outer similarity of the streamwise velocity statistics in the outer layer of fully developed 
turbulent channel flow over two very different roughness topologies. As with the study of Antonia \& Krogstad (2001), roughness topologies were tested with similar $u_{\tau}$ and $k$, but with $k / \delta$ exceeding the Jiménez limit so that significant inner-outer interaction is to be expected. In the first instance, it is not clear how large the roughness should be in order for the log law to be invalid. In the second instance, it is by no means clear the extent to which information from a rough surface propagates to the outer region such that outer similarity (or $u_{\tau}, \delta$ scaling) also fails. For sufficiently large roughness of course, it is likely that the entire boundary layer will be engulfed by the effects of the roughness.

\section{Experimental details}

\subsection{Flow facility}

Experiments were carried out in a purpose-built rough-wall channel facility. The driving pressure is provided by a $4.7 \mathrm{~m}^{3} \mathrm{~s}^{-1}$ squirrel-cage blower powered by a computer-controlled, variable-speed $\mathrm{AC}$ motor, and the flow is conditioned by a $10 \mathrm{~mm}$ hexagonal honeycomb, a series of three $0.5 \mathrm{~mm}$ screens and an 8:1 twodimensional contraction. The blower and motor are mounted on vibration-isolating pads, and are coupled to the flow-conditioning section with a flexible neoprene membrane to minimize the transmission of mechanical vibrations. A short adaptor section directs the flow from the contraction into the channel, and is lined with grit roughness in order to trip the wall boundary layers and accelerate flow development.

The channel consists of a series of modular rail-mounted sections, each with an internal baseline half-height, $h=50.8 \mathrm{~mm}$ (measured from the lowest point of the roughness), width $W=15 h$ and length $L=24 h$. Special care was taken in the assembly and alignment of the sections to ensure a continuous and parallel interior surface. For all the present measurements, five such sections were used for a total fetch of $134 h$ between the adaptor section inlet and the measurement station. An instrumented channel section was fitted with an externally mounted, computer-controlled three-axis traverse having a resolution of $5 \mu \mathrm{m}$ and a range of $2 h$ along the spanwise and wallnormal axes. The channel sections could be re-arranged to position the instrumented test section anywhere along its streamwise fetch, and the test section was fitted with multiple ports capable of receiving the traverse. A series of 11 static pressure ports were installed equidistantly along one side wall, and a Pitot tube was positioned at the channel centreline $2 h$ upstream of the channel exit to measure local mean velocity. A flow temperature sensor was also positioned in the channel upstream of the exit.

Roughness was applied to the upper and lower wetted surfaces of the channel only. No roughness could be applied to the channel sides owing to interference with the channel instrumentation. The effect of the reduced wall shear of the smooth sidewalls was tested, and the sidewall condition was found to have no observable effect upon the velocity statistics within $2 h$ of the channel centreline up to the fourth order. The resulting underprediction of wall shear was found to be within the bounds of the overall experimental uncertainty. Discontinuities in the roughness patterns were present between the channel sections, but care was taken to minimize them.

\subsection{Roughness topologies}

A grit-type roughness, a mesh-type roughness and a smooth surface were tested. The grit-type roughness is a 16-gauge industrial open-type silicon carbide abrasive sheet with a sparse and isotropic grit pattern having a highly non-Gaussian distribution, thereby precluding the behaviour usually associated with highly uniform 

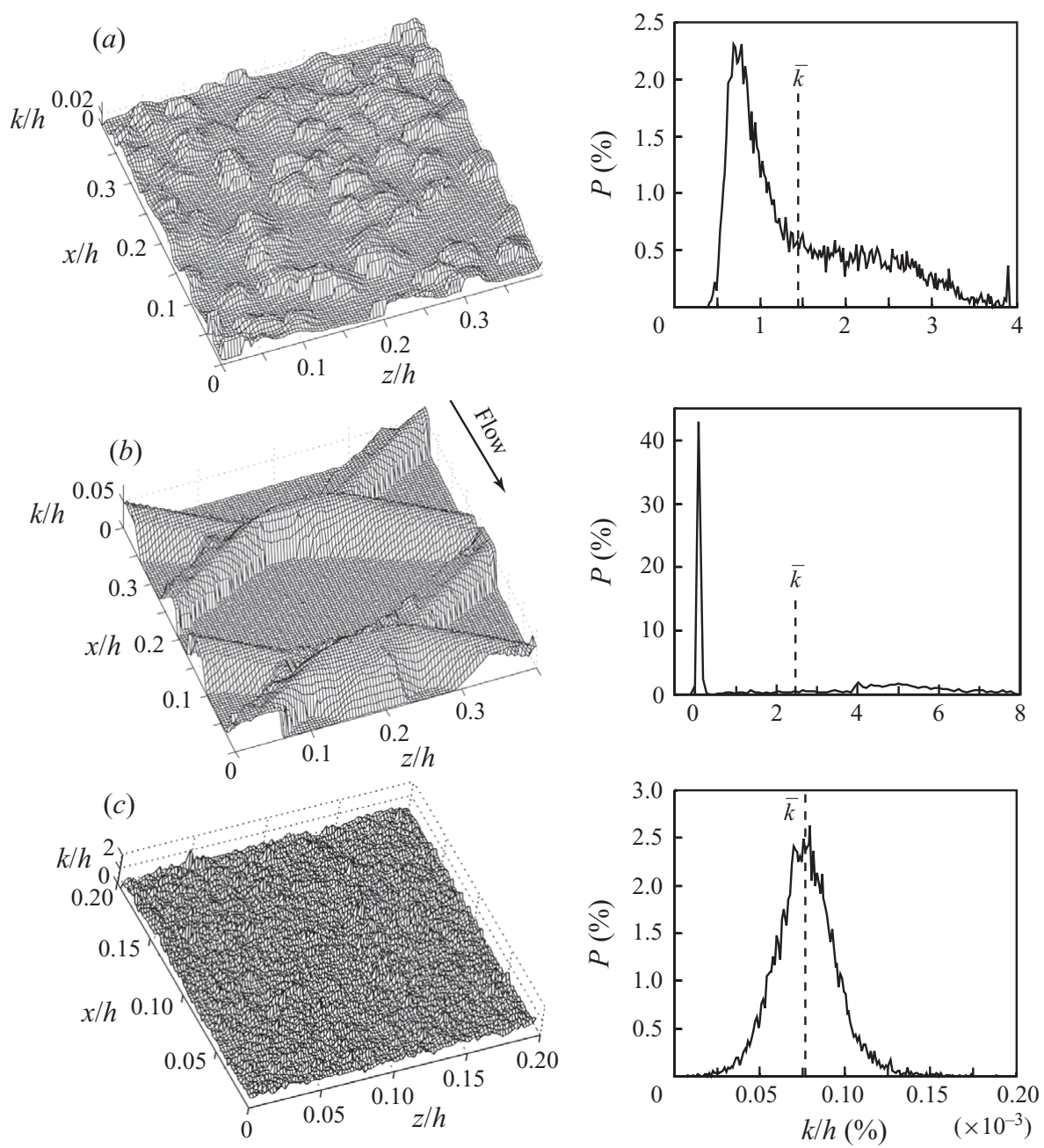

FIGURE 1. Laser profilometer results and roughness height distributions for the $(a)$ grit roughness, $(b)$ mesh roughness and $(c)$ smooth surfaces.

grit (Nikuradse 1933). The mesh-type roughness used was an expanded aluminium sheet consisting of twisted $2.36 \times 1.6 \mathrm{~mm}$ rectangular elements forming a diamondshaped pattern with a centreline spanwise-to-lengthwise aspect ratio, $L_{z} / L_{x}=2.6$. The mesh was oriented such that the downstream edges of individual elements protruded further into the flow than the upstream edges. The smooth surface was a sheet of wood which was sanded and finished with care. The topologies of all three surfaces were digitized using a laser profilometer (with a measurement resolution of $1 \mu \mathrm{m}$ ), and the resulting surface maps and roughness-height probability distribution functions, $P(k)$, are included in figure 1 .

The key roughness parameters for the three surfaces are also included in table 1 . As noted by Colebrook \& White (1937), the larger roughness elements exert a disproportionately large effect because the pressure drop across a particular element is proportional to $k^{2}$. They also showed how the larger elements 'shield' the smaller ones. Owing to the necessity of describing a roughness topology using a single, characteristic roughness length scale, the maximum roughness height (equivalent to 


$\begin{array}{lccccc}\text { Surface } & k_{\max }(\mathrm{mm}) & \bar{k}(\mathrm{~mm}) & k_{r m s}(\mathrm{~mm}) & \bar{k} / h(\%) & k_{\max } / h(\%) \\ \text { Grit } & 2.0 & 0.74 & 0.41 & 1.46 & 3.94 \\ \text { Mesh } & 4.0 & 1.27 & 1.31 & 2.50 & 7.87 \\ \text { Smooth } & 0.095 & 0.039 & 0.0093 & 0.077 & 0.19\end{array}$

TABLE 1. Key surface parameters.

the width of the roughness distributions), $k_{\max }$, is used. Therefore, the rough surfaces are subsequently compared using $k_{\max }$, and by inference, the equations of $\S 1 \mathrm{imply}$ $k=k_{\max }$. In the case of the mesh, $L_{z} / k_{\max }=7.5$ and $L_{x} / k_{\max }=2.9$. In the case of the grit roughness, the plan area coverage density, $\lambda_{p}$ (Leonardi \& Castro 2010), varies rapidly with $y$ and is therefore not a useful parameter. In the case of the mesh roughness, however, $\lambda_{p} \approx 50 \%$.

\subsection{Data acquisition and reduction}

Time-domain $u$-component velocity measurements were made using $5 \mu \mathrm{m}$ diameter platinum hot-wire probes with sensing length, $0.5 \leqslant l \leqslant 1.0 \mathrm{~mm}$, yielding a nominal length-to-diameter ratio of $100 \leqslant l / d \leqslant 200$. The effect of the stub length, $L$, can be significant: in the present case, $L / l \approx 1$, and Li et al. (2004) show that at these quite large ratios, any effects of conduction increase very slowly as $l / d$ decreases. No compensation was made for the effects of spatial resolution where $41 \lesssim l^{+} \lesssim 62$. The error in $U$ arising from the sensitivity of the single-sensor hot-wire to wall-normal fluctuations was estimated at less than $5 \%$ at the location of peak $\overline{u^{2}}$ for all cases; this was further verified by comparing selected cases to measurements taken with a cross-wire probe. The directionally resolved peak values of $\overline{u^{2}}$ agreed with the single-sensor measurements to within less than $5 \%$ (these results shall be reported in a subsequent publication). The sensors were driven by in-house monolithic hotwire anemometers with an overheat ratio of 0.8 . The anemometers have a typical frequency response of $10 \mathrm{kHz}$, and the high-frequency roll-off was shown to have no significant effect on the measured velocity moments up to the fourth order. The system is described in detail by Birch \& Morrison (2010). The sensors were calibrated in situ against the mean channel centreline velocity, and were automatically recalibrated throughout each experiment at approximately half-hour intervals. The linearization error arising from the unsteady reference flow was estimated according to the method of Breuer (1995) and was determined to be typically less than $0.6 \%$. Velocity statistics were collected at a single spanwise location unless otherwise indicated. For the case of the grit, measurements were made at the closest point to the channel centreline where there was a local region of small $k$. For the case of the mesh, measurements were made close to the centre of a depression. In this manner, statistics were obtained from $y<k_{\max }$ through to $y \geqslant h$.

Flow temperature was monitored continuously throughout the experiments using a PTC thermistor probe, and data were discarded if any two successive calibration curves varied by more than $2 \%$. No temperature corrections were applied. Anemometer signals were amplified using a TL074CN low-noise operational amplifier (with a typical slew rate of $13 \mathrm{~V} \mathrm{\mu s}^{-1}$ ) and were low-pass RC filtered at $100 \mathrm{kHz}$ prior to digitization. Signals were collected and digitized using a Data Translation DT9836 16 bit data acquisition card with a full-scale range of $\pm 10 \mathrm{~V}$. Channels were sampled simultaneously at $60 \mathrm{kHz}$, and $2^{22}$ data points were collected per channel (unless otherwise indicated). The estimated error bounds on the experimental results are 


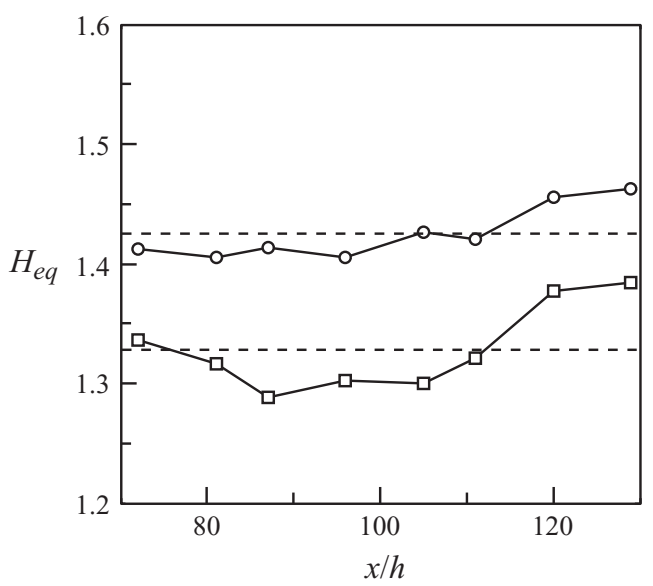

FIGURE 2. Streamwise variation of $H_{e q}(3.1)$; $\bigcirc$, mesh; $\square$, grit. Dashed lines indicate mean values.

as follows: $u_{\tau}, 3 \%$; mean velocities, $0.5 \%$; second-order moments, $1.5 \%$; velocity skewness and kurtosis, $6 \%$ and $10 \%$, respectively.

\section{Results}

\subsection{Flow validation}

Wall-normal velocity traverses were performed for $72 \leqslant x / h \leqslant 129$ over both types of roughnesses in order to ensure that the turbulence was fully developed. In order to compare the shape of the velocity profiles at the various stations independently of the near-wall scaling parameters (which are subject to a much greater experimental uncertainty), an equivalent shape factor $H_{e q}(x)$ was defined as

$$
H_{e q}(x)=\frac{\int_{y_{1}(x)}^{y_{2}(x)}\left(1-\frac{U}{U_{c l}}\right) \mathrm{d} y}{\int_{y_{1}(x)}^{y_{2}(x)}\left(\frac{U}{U_{c l}}\right)\left(1-\frac{U}{U_{c l}}\right) \mathrm{d} y},
$$

where $U_{c l}$ is streamwise-invariant, and $y_{1}(x)$ and $y_{2}(x)$ are selected such that $U\left(y_{1}\right) / U_{c l}$ and $U\left(y_{2}\right) / U_{c l}$ are equal to 0.05 and 0.95 , respectively. Normalizing with $U_{c l}$ also ensures that this shape factor is unaffected by any uncertainty in $u_{\tau}$. The variation of $H_{e q}$ with streamwise distance is found to be less than $\pm 2 \%$, which is within the range of experimental error (figure 2), indicating that, according to this definition, the flow was fully developed well upstream of the $x / h=128$ measurement station. The streamwise variations in the skewness $\overline{u^{3}}$ and kurtosis $\overline{u^{4}}$ profiles were found to be within \pm 3 and $\pm 5 \%$, respectively, over $72 \leqslant x / h \leqslant 129$ for both the grit and mesh roughness.

The two-dimensionality of the mean flow was also verified at $x / h=128$ by examining the mean and r.m.s. velocity distributions in both the wall-normal and spanwise directions. Figure 3 shows the mean and r.m.s. velocities over the larger mesh-type roughness as functions of spanwise position at $y / h=0.14,0.28,0.40$, 0.60 and 0.80 . The variation increases slightly with increasing wall proximity, owing to the inhomogeneities introduced by the individual roughness elements; this effect is 

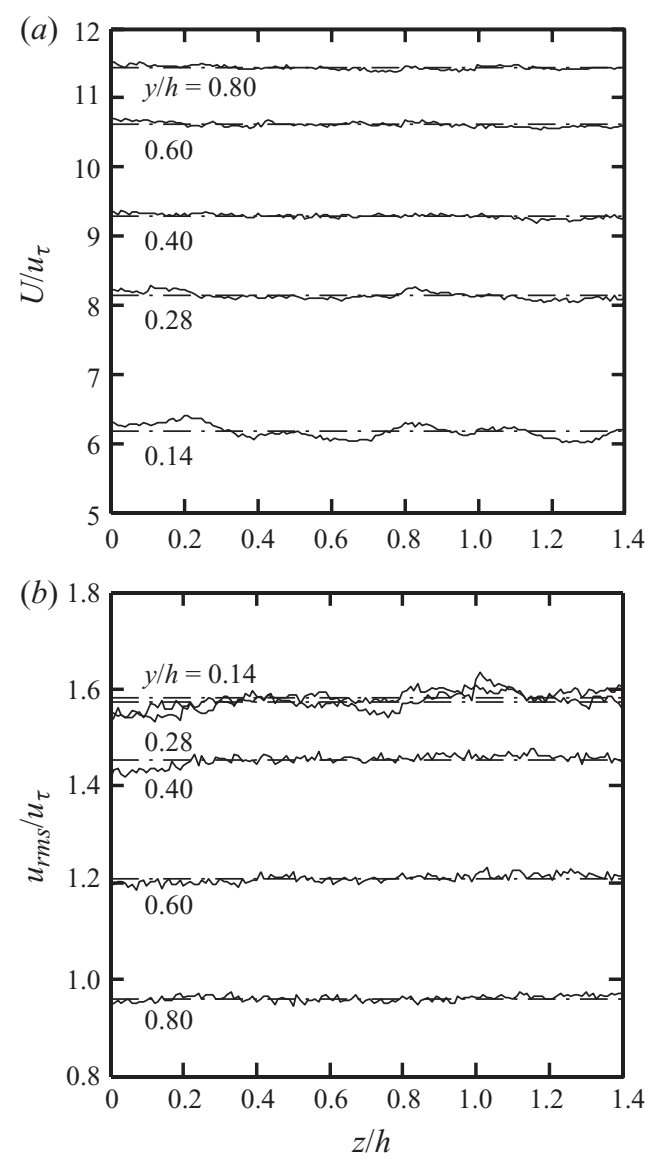

FIGURE 3. Spanwise variation in $(a)$ mean velocity and $(b)$ r.m.s. velocity over the mesh roughness.

more pronounced over the anisotropic and periodic mesh. The maximum variations are plotted as a function of wall-normal distance in figure 4, showing convergence to within less than $5 \%$ for all quantities, for $y / h>0.14$. Also, $H_{e q}$ was computed at several spanwise locations at $x / h=128$, and was found to vary by less than $1 \%$ from the overall channel mean.

The symmetry of the flow about the centreline was also verified at $x / h=128$. Mean and r.m.s. velocity profiles collected over the lower and upper wetted surfaces were found to collapse to within $2.5 \%$ in the range $0.13<y / 2 h<0.87$ for both roughness types. For values of $y / 2 h$ outside this range, the flow was affected locally by the wakes of the individual roughness elements, which were not necessarily symmetrically arranged. Mean velocity profiles at $x / h=128$ were also obtained over both roughness types using a Pitot tube and a differential pressure transducer (with a nominal sensitivity of $1 \mathrm{mV} \mathrm{Pa}^{-1}$ ). These mean velocity profiles were compared with those from the hot wire and found to agree to within $0.5 \%$ for $y / h>0.25$. The mean velocity profiles could not be reliably compared closer to the surface, owing to the uncertainty in the position of a sensor relative to the local roughness elements and the much larger size of the Pitot tube. 


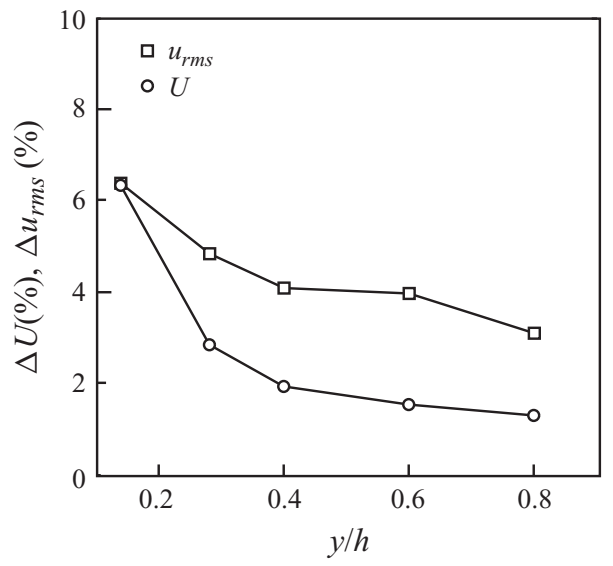

FIGURE 4. Maximum spanwise variation in $(a)$ mean velocity and $(b)$ r.m.s. velocity over the mesh-type roughness. Symbols: $\bigcirc, \Delta U ; \square, \Delta u_{r m s}$.

\subsection{Determination of flow scaling parameters}

In fully developed channel flow, the wall friction is proportional to the streamwise pressure gradient $\mathrm{d} p / \mathrm{d} x$, and the coefficient of friction, $C_{f}$, may be expressed as

$$
C_{f}=2\left(\frac{u_{\tau}}{U_{c l}}\right)^{2}=-\frac{h}{\frac{1}{2} \rho U_{c l}^{2}} \frac{\mathrm{d} p}{\mathrm{~d} x}
$$

and is independent of both fetch and Reynolds number. Note that $C_{f}$ values for the grit and mesh roughness were calculated over the range $4 \times 10^{4}<R e_{h}<9 \times 10^{4}$, where $\mathrm{d} p / \mathrm{d} x$ was estimated by a linear regression of side wall pressure measurements for $112 \leqslant x / h \leqslant 132$.

The exact form of the friction factor relationship in smooth pipe flow has recently been reassessed (see McKeon, Zagarola \& Smits 2005), but noting that differences at moderate Reynolds numbers are imperceptibly different from Prandtl's 'universal friction factor relationship' given by

$$
\frac{1}{\sqrt{\lambda}}=2.0 \log _{10}\left(\frac{\bar{U} D}{v} \sqrt{\lambda}\right)-0.8
$$

we use (3.3) to estimate $C_{f}$ in the smooth channel. Here $\lambda=8\left(u_{\tau} / \bar{U}\right), \quad D$ is the pipe diameter and $\bar{U}$ is the bulk-mean velocity. Taking the effective hydraulic diameter of the channel as $D_{h}=4 h W /(2 h+W)$, (3.3) may be used to estimate $\lambda$ with $D=D_{h}$ and $C_{f}$ calculated as

$$
C_{f}=\frac{h}{D_{h}}\left(\frac{\bar{U}}{U_{c l}}\right)^{2} \lambda .
$$

Figure 5 shows measurements of $C_{f}$ for the two rough surfaces together with those for the smooth channel. In the case of the rough surfaces, the variation of $C_{f}$ with $R e_{h}$ is less than $1 \%$ in both cases. For the smooth surface, the results agree well with (3.3), and, in what follows, a value of $C_{f}=2.62 \times 10^{-3}$ is used for the smooth wall at $R e_{h}=12.4 \times 10^{4}$. 


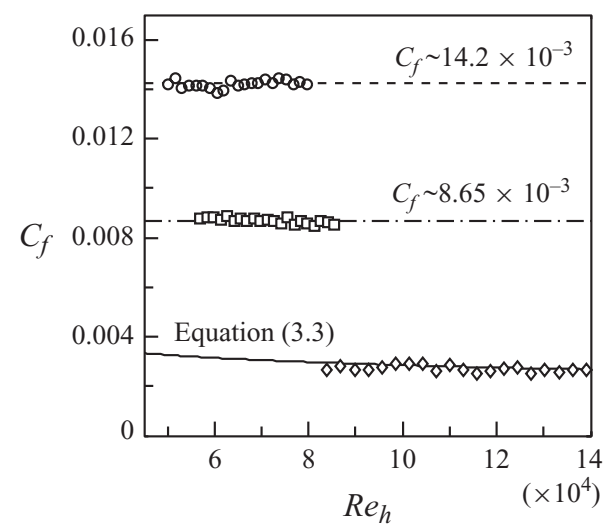

FIGURE 5. Variation in the coefficient of friction with Reynolds number: $\bigcirc$, mesh roughness; $\square$, grit roughness; $\diamond$, smooth surface.

The relative roughness, $k_{s} / h$, was also calculated using Nikuradse's law for fully rough pipes,

$$
\frac{1}{\lambda^{1 / 2}}=2 \log _{10}\left(3.71 \frac{D}{k_{s}}\right),
$$

with $D=D_{h}$. The values of $k_{s} / h$ were found to be, respectively, 4.6 and $18.4 \%$ for the grit and mesh roughness; these are within the scope of those included in the study by Wu \& Christensen (2007).

The zero-plane displacement, $d$, and the roughness length, $y_{0}$, defined by (1.2) are not amenable to direct measurement. To preserve generality and accommodate the significant degree of uncertainty in the absolute position of the probe relative to the channel wall, both were estimated using (1.2): it was assumed that a logarithmic region exists and that it begins at the wall-normal location at which the mean velocity first exhibits spanwise independence, taking this as an indication that the mean velocity is no longer influenced by the near-wall inhomogeneities. Since the flow over the rough surfaces has already been shown to be fully rough and fully developed, it was further assumed that $y_{0}$ is a property of the surface geometry only. Values of $y_{0}$ and $d$ for each of the data sets were then obtained by fitting the mean velocity profiles to (1.2) with $\kappa=0.41$. The uncertainty in $y_{0}$ was estimated to be less than $12 \%$ : a variation in $\kappa$ by \pm 0.01 resulted in a variation in $y_{0}$ and $d$, which was within the range of the overall experimental error. The method for estimating $y_{0}$ and $d$ is described in the Appendix. Table 2 shows the critical parameters for the data sets presented.

\subsection{Mean flow scaling}

Figure 6 shows the viscous-scaled mean velocity profiles over the mesh and grit roughness and the smooth surface at $x / h=128$. Selected data from similar experiments in channels with rough walls (Bakken et al. 2005) and smooth walls (Monty 2005) are also presented. The relevant experimental parameters, as well as a description of the symbols and lines used in the plots, are listed in table 3. It is clear from the present smooth-wall data (in particular, the very large wake component), that the flow is not fully developed. In contrast, the results of Monty (2005) (taken at a streamwise fetch $x / h>350$ ) do show full development, and for these data Monty deduces $\kappa=0.384$ and $B=4.33$. However, for a consistent comparison with the present rough-wall data, here we take $\kappa=0.41$ for which $B=5.0$. 


\begin{tabular}{lccccccccc}
\hline Case & $U_{c l}\left(\mathrm{~m} \mathrm{~s}^{-1}\right)$ & $U_{c l}^{+}$ & $\bar{U}\left(\mathrm{~m} \mathrm{~s}^{-1}\right)$ & $R e_{\tau}$ & $R e_{h} \times 10^{-4}$ & $d(\mathrm{~mm})$ & $y_{0}(\mathrm{~mm})$ & $k^{+}$ & $k_{s}^{+}$ \\
Grit (G1) & 24.7 & 15.2 & 21.0 & 4780 & 7.28 & 1.35 & 0.13 & 186 & 221 \\
Grit (G2) & 26.7 & 15.2 & 22.8 & 5130 & 7.78 & 1.30 & 0.13 & 200 & 238 \\
Grit (G3) & 28.6 & 15.2 & 24.4 & 5540 & 8.43 & 1.45 & 0.13 & 216 & 257 \\
Mesh (M1) & 21.1 & 11.8 & 17.3 & 5230 & 6.15 & 1.90 & 0.43 & 410 & 960 \\
Mesh (M2) & 23.2 & 11.6 & 18.8 & 5830 & 6.77 & 2.60 & 0.43 & 458 & 1070 \\
Mesh (M3) & 25.0 & 11.8 & 20.5 & 6270 & 7.38 & 2.00 & 0.43 & 492 & 1150 \\
Smooth (S) & 41.9 & 29.6 & 37.4 & 4477 & 12.4 & 0.75 & 0.0015 & 8.37 & - \\
Monty (2005) & 30.8 & 25.7 & 28.1 & 3947 & 10.1 & - & - & - & -
\end{tabular}

TABLE 2. Experimental parameters for the grit and mesh roughnesses. Here $k^{+}$is based on $k_{\max }$.

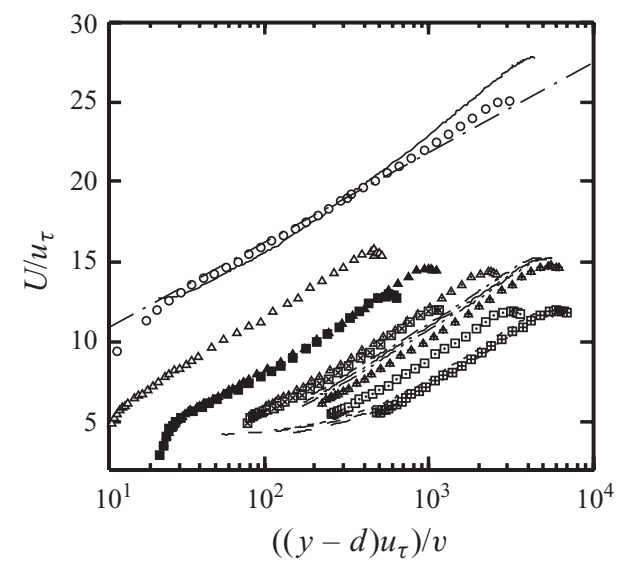

FIGURE 6. Viscous-scaled mean velocity profiles. Data from Bakken et al. (2005) are included for comparison; see table 3 for description of symbols and lines. Dash-dotted $\left(-\cdot-^{-}\right),(1.1)$ with $\kappa=0.41, B=5.0$ and $\Delta U^{+}=0$.

A direct comparison between smooth- and rough-wall mean velocity profiles at comparable Reynolds numbers may be made by examining the values of the Hama (1954) roughness function, $\Delta U^{+}$, obtained from (1.3), and taking $B=5.0$. The velocity shifts shown in figure 6 are tabulated in table 4: these indicate some small $R e$ dependence. It is significant to note that the values of $\Delta U^{+}$for the present mesh and grit roughness surfaces agree reasonably well with those for the $R e_{\tau}=6000$ rod roughness and $R e_{\tau}=5600$ mesh roughness of Bakken et al. (2005), respectively, although the present profiles extend to much smaller values of $y^{+}$. A second estimate of $\Delta U^{+}$may be obtained from the friction coefficients, as

$$
\Delta U^{+}=\sqrt{\frac{2}{C_{f_{0}}}}-\sqrt{\frac{2}{C_{f}}},
$$

where $C_{f_{0}}$ is the smooth-wall coefficient of friction (table 4): there are significant differences between estimates for the two roughness types. Equation (3.6) tends to slightly overpredict $\Delta U^{+}$.

Figure $7(a)$ shows mean velocity profiles with inner scaling, $\left(u_{\tau}, y_{0}\right)$. A small logarithmic region is observed in both profiles, extending from $15 \lesssim(y-d) / y_{0} \lesssim 30$, as well as an extensive wake region. Estimates of the wake strength (the vertical 


\begin{tabular}{|c|c|c|c|c|}
\hline Symbol & Source & Roughness & $R e_{\tau}$ & $R e_{k}$ \\
\hline$-\cdot-\cdot$ & Present study & Grit (G1) & 4780 & 186 \\
\hline$-\cdot-\cdot$ & Present study & Grit (G2) & 5130 & 200 \\
\hline$-\cdots-\cdot \cdot$ & Present study & Grit (G3) & 5540 & 216 \\
\hline$-\ldots$ & Present study & Mesh (M1) & 5230 & 410 \\
\hline--- & Present study & Mesh (M2) & 5830 & 458 \\
\hline-- & Present study & Mesh (M3) & 6270 & 493 \\
\hline & Present study & Smooth & 4477 & - \\
\hline$\bigcirc$ & Monty (2005) & Smooth & 3947 & - \\
\hline 0 & Bakken et al. (2005) & Smooth & 670 & - \\
\hline$\odot$ & Bakken et al. (2005) & Smooth & 3300 & - \\
\hline$\triangle$ & Bakken et al. (2005) & Mesh & 450 & 15 \\
\hline$\Delta$ & Bakken et al. (2005) & Mesh & 950 & 32 \\
\hline$\Delta$ & Bakken et al. (2005) & Mesh & 2500 & 83 \\
\hline$\triangle$ & Bakken et al. (2005) & Mesh & 5600 & 187 \\
\hline $\mathbf{\square}$ & Bakken et al. (2005) & Rod & 600 & 20 \\
\hline$\otimes$ & Bakken et al. (2005) & Rod & 1200 & 40 \\
\hline$\bullet$ & Bakken et al. (2005) & Rod & 3200 & 107 \\
\hline$\boxplus$ & Bakken et al. (2005) & Rod & 6000 & 200 \\
\hline$\diamond$ & Flack et al. (2005) & Grit and mesh & $\sim 6200$ & $60 \sim 120$ \\
\hline-- & Jiménez (2004) & Various & $\geq 6000$ & - \\
\hline
\end{tabular}

TABLE 3. Symbols used in plots, unless otherwise specified.

$\begin{array}{lccccc}\text { Case } & R e_{\tau} & y_{0}^{+} & \Delta U^{+},(1.3) & \Delta U^{+},(3.6) & \Delta U_{\text {wake }}^{+} \\ \text {G1 } & 4780 & 12.23 & 11.1 & 12.4 & 0.68 \\ \text { G2 } & 5130 & 13.13 & 11.3 & 12.4 & 0.78 \\ \text { G3 } & 5540 & 14.17 & 11.5 & 12.4 & 0.81 \\ \text { M1 } & 5230 & 44.26 & 14.2 & 15.8 & 0.45 \\ \text { M2 } & 5830 & 49.39 & 14.5 & 15.8 & 0.19 \\ \text { M3 } & 6270 & 53.10 & 14.7 & 15.8 & 0.29 \\ \text { S } & 4477 & 0.13 & - & - & 2.48 \\ \text { Monty (2005) } & 3947 & - & - & - & 0.50\end{array}$

TABLE 4. Estimates of the Hama roughness function, $\Delta U^{+}$, and wake strength, $\Delta U_{\text {wake }}^{+}$.

separation between the maximum ordinate value and the extrapolated log law at the same value of $\left.(y-d) / y_{0}\right)$ may be made and compared with the equivalent wake strength for the smooth channel (figure 6): estimates for the wake strength, $\Delta U_{\text {wake }}^{+}$, are calculated as

$$
\Delta U_{\text {wake }}^{+}=U^{+}-\frac{1}{\kappa} \ln \left(y_{c l}-d\right)^{+}-B+\Delta U^{+},
$$

where $y_{c l}$ is the location of maximum $U^{+}$, and $\Delta U^{+}$is given by (1.3). It is clear that for the grit surface, $\Delta U_{\text {wake }}^{+}$increases slightly, while that for the mesh surface decreases compared with the smooth-wall value. There are also some slight variations in $\Delta U_{\text {wake }}^{+}$with $k^{+}$, but in the case of the mesh roughness, there is no discernible trend.

The outer-scaled velocity deficit profiles are shown in figure $7(b)$ : the profiles over the grit surface agree very well with the mesh data from Bakken et al. (2005); for clarity, these are not shown. For $(y-d) / h \lesssim 0.06$, the present data are well 


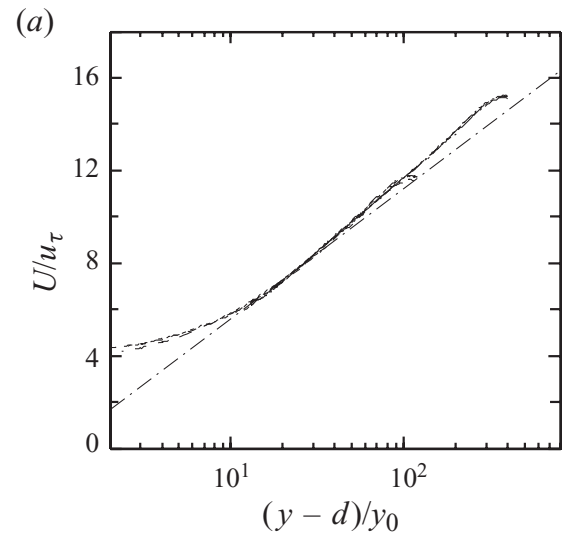

(b)

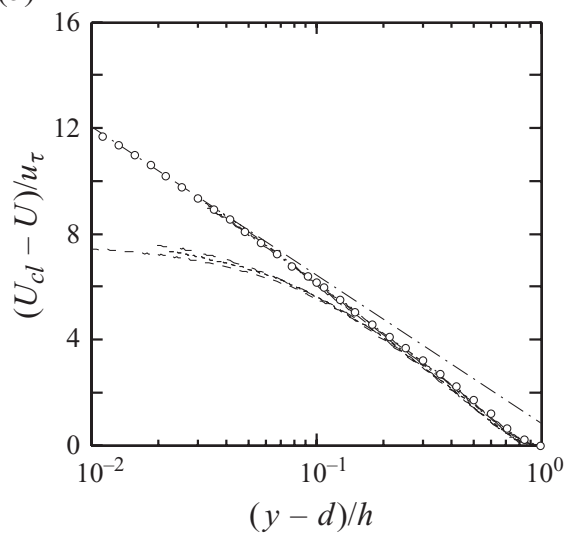

FIGURE 7. Mean velocity profiles: $(a)$ inner scaling, $U^{+}$versus $\ln \left((y-d) / y_{0}\right) ;(-\cdot-\cdot),(1.2)$.

(b) Outer scaling $\left(U_{c l}-\bar{U}\right)^{+}$versus $\ln ((y-d) / h) ;(-\cdot-\cdot),(3.8)$.

represented by

$$
\frac{U_{c l}-U}{u_{\tau}}=-\frac{1}{0.41} \ln \left(\frac{y-d}{h}\right)+0.8,
$$

where the additive constant is adjusted to the data. Significantly, the profiles over the mesh surface do not collapse $(y-d) / h \lesssim 0.30$. These data were recorded for a spanwise location coincident with the centre of the depression ('trough'); however, the spanwise wavelength of the mesh is much larger than the roughness length scale. Therefore, a further 24 profiles were collected across a single roughness wavelength in order to assess the effect of spanwise inhomogeneities. Owing to mechanical interference, the wall-normal extent of the traverses at crest locations (figure 8) was restricted to $y>k$. Figure 8 shows the mean (time-averaged) velocity profiles scaled against outer variables for trough and crest locations together with one spatially averaged over a wavelength. They collapse (become spanwise homogeneous) for $(y-d) / h \gtrsim 0.08$ only $(y / k>1.65)$, that is, at a wall-normal distance less than that at which outer scaling given by (3.8) appears. It therefore appears that the lack of collapse is not a direct consequence of the mesh spanwise periodicity. This phenomenon was not observable in the results of Bakken et al. (2005), as those data did not extend to sufficiently small $(y-d) / h$. 


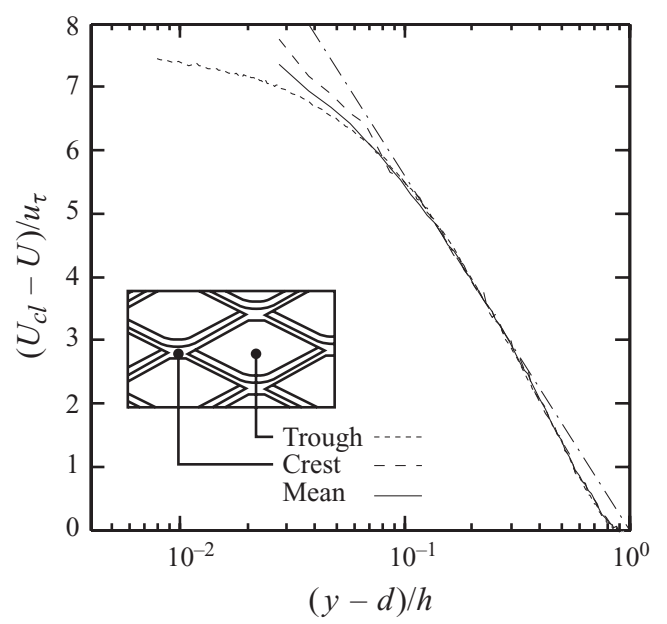

FIGURE 8. Spanwise variation of the mean velocity profiles over the mesh-type roughness at $R e_{\tau}=5890$.

The existence of a self-similar log region necessarily requires simultaneous collapse with inner and outer scaling in order to demonstrate independence from both. In the case of both surfaces, collapse on inner scales for $15 \lesssim(y-d) / y_{0} \lesssim 30$ is apparent: for the grit surface, this is equivalent to $0.038 \lesssim(y-d) / h \lesssim 0.076$ and there appears to be simultaneous collapse for $0.04 \lesssim(y-d) / h \lesssim 0.06$. However, in the case of the mesh surface, collapse on inner scales is equivalent to $0.127 \lesssim(y-d) / h \lesssim 0.254$ but it has already been noted that collapse on outer scales does not occur for $(y-d) / h \lesssim 0.30$. This therefore precludes self-similarity.

For there to be a log law in the forms given by (1.2) and (1.4), the ratio of velocity scales $\xi=\left(U_{c l}-\bar{U}\right)^{+}$must be independent of the Reynolds number (Zagarola \& Smits 1998). This is readily deduced from (1.4) when at sufficiently high Reynolds number, $B^{*}$ tends to a constant. Figure 9 shows $\xi$ plotted as a function of $R e_{\tau}$ for both rough surfaces. As the foregoing suggests, estimates for the mesh surface show slightly greater variation than those for the grit surface. In fully developed smooth pipe flow, McKeon et al. (2004) suggest a value of $\xi=4.28$. In their rough channel, Bakken et al. (2005) suggest a value of $\xi=2.64$. The present data suggest $\xi=2.22$ and 2.14 for the grit and mesh surfaces, respectively. Except for the mesh surface for which self-similarity has not been clearly demonstrated, the different values of $\xi$ are due to the different boundary conditions in each experiment and are not themselves an indication of a lack of self-similarity. In particular, the additive constant in the log law is strongly dependent on the surface roughness, even when it is fully rough.

\subsection{Scaling of turbulence statistics}

Figure 10 shows $u$-component second-order moments scaled with viscous, inner and outer scales. Also shown for viscous and outer scaling are the smooth-wall results of Monty (2005), as well as selected data from Bakken et al. (2005), mesh and rod data in the range $15 \leqslant R e_{k} \leqslant 200$, which is at lower Reynolds numbers than those of the present data. All data in figure 10(a) show a suppression of $\overline{u^{2}}$ near the wall: the locus of the maximum does not scale with $v / u_{\tau}, y_{0}$ or $h$. Interestingly, the maxima for the mesh data are lower than those for the grit data even though the mesh maximum height is larger. The inner-scaled $\overline{u^{2}}$ profiles (figure $10 b$ ) collapse remarkably well for 


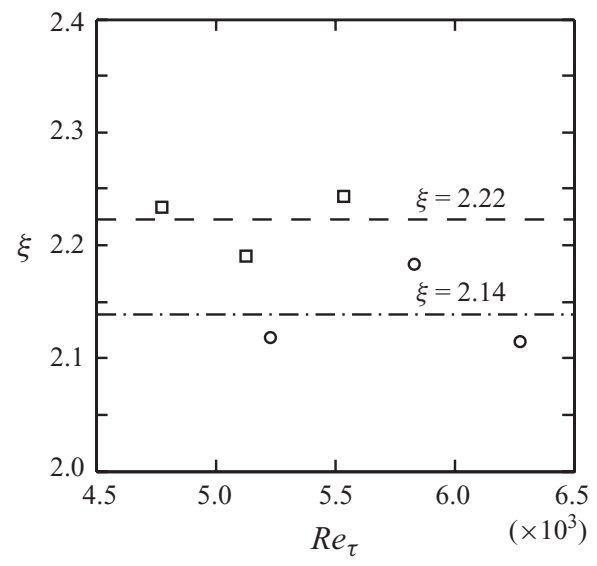

FIGURE 9. $\xi$ as a function of $R e_{\tau}: \bigcirc$, mesh-type roughness; $\square$, grit-type roughness.

$(y-d) / y_{0} \gtrsim 30$ and 50 (or, equivalently, $(y-d) / h \gtrsim 0.25$ and 0.13 ) for the mesh and grit roughnesses, respectively.

Figure $10(c)$ shows the outer-scaled profiles of $\overline{u^{2}}$ from the present study, together with selected data from Monty (2005), Bakken et al. (2005) and data reviewed by Jiménez (2004). The results of Bakken et al. (2005) collapse into two families of curves for $y / h \lesssim 0.4$; they attribute this bifurcation to a Reynolds number effect, as the mode selection seemed to be determined by whether or not the equality $R e_{\tau}<1200$ was satisfied. It is curious that, although the data of Monty (2005) (for which $R e_{\tau}=3947$ ) and those reviewed by Jiménez (2004) (for which all are $R e_{\tau}>6000$ ) collapse well with the high- $R e_{\tau}$ curve of Bakken et al. (2005), the present high- $R e_{\tau}$ data collapse with the low- $R e_{\tau}$ curve. Similar behaviour appears in the third-order moments. Figure 11 shows a surface-independent collapse of all the present data for $(y-d) / h \gtrsim 0.15$, which is a lower value than all of the data indicated by figure $10(c),(y-d) / h \gtrsim 0.45$, suggesting that Townsend's outer similarity is remarkably robust. Note that, in the case of figure $11, k / h$ for the present roughness (grit, $4 \%$; mesh, $8 \%$ ) is larger than the limit of $2.5 \%$ suggested by Jiménez (2004).

Figure 12 shows the viscous-, inner- and outer-scaled third-order moments, $\overline{u^{3}}$. The viscous-scaled profiles (figure 12a) indicate again the good agreement with the results of Bakken et al. (2005). The inner-scaled profiles (figure 12b) demonstrate the good convergence of the present data, though the profiles exhibit some $R e$-dependence and collapse only over the half-decade of $(y-d) / y_{0}$ closest to the channel centreline. With outer scaling, $\bar{u}^{3}$ collapses for both surfaces for $(y-d) / h \gtrsim 0.6$.

Figure 13 shows profiles of the fourth-order moment, $\overline{u^{4}}$, scaled with inner and outer variables. Convergence of the fourth-order moments was verified by comparing the values at selected wall-normal locations to averages sampled over a time $t^{+}=t u_{\tau}^{2} / v=3.0 \times 10^{8}$ and $3.8 \times 10^{8}$ for the grit and mesh surfaces, respectively. The inner-scaled $\overline{u^{4}}$ profiles collapse well and, unlike the profiles of $\overline{u^{3}}$, show no clear Reynolds number dependence. The outer-scaled profiles also collapse well for $(y-d) / h \gtrsim 0.15$.

Since only the odd-order moments are sensitive to the sign of $u$, the improved collapse of the even-order velocity moments was further investigated by considering 

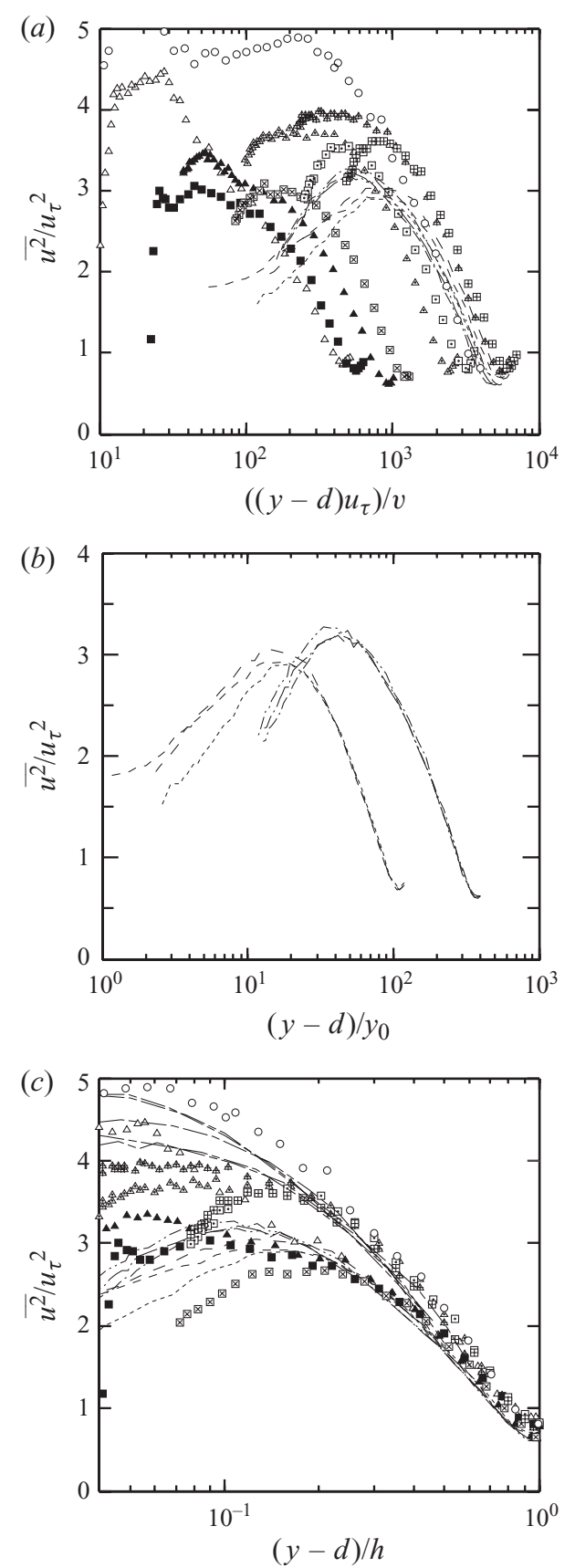

FIGURE 10. Second moment ${\overline{u^{2}}}^{+}:(a)$ viscous scaling, $(b)$ inner scaling and $(c)$ outer scaling.

the wall-normal distribution of the time-mean Heaviside function,

$$
\bar{H}(y)=\frac{1}{t} \int_{0}^{t} H(u(y)) \mathrm{d} t .
$$




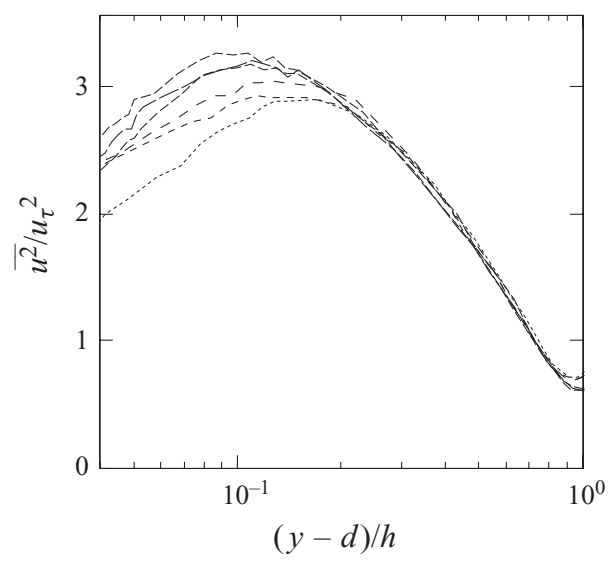

FIGURE 11. Outer-scaled second moment ${\overline{u^{2}}}^{+}$.

The Heaviside function provides a robust, quantitative measure of the distribution of positive and negative velocity perturbations, which is independent of magnitude (it should be noted that for statistically stationary, homogeneous isotropic turbulence, $\bar{H}=0.5$ ). Figure 14 shows the variation of $1-\bar{H}$ over the mesh and grit surfaces at $R e_{\tau}=5830$ and 5130, respectively. As with the third-order moment (and, to a lesser extent, the outer-scaled mean velocity), the curves diverge for $(y-d) / h \lesssim 0.4$. At small wall-normal distances, velocity perturbations over the mesh roughness are more likely to be negative than over the grit roughness. The collapse of $\overline{u^{2}} / u_{\tau}^{2}$ for $(y-d) / h \gtrsim 0.15$ indicates that the normalized mean magnitudes of the perturbations are similar. Together, these results suggest that the probability of momentum transport away from the wall by larger structures is weakly dependent on the surface type.

\subsection{Space and time velocity correlations}

Figure 15 shows the $u$-component auto-correlation function

$$
R_{11}(\boldsymbol{x} ; \Delta t)=\frac{\overline{u(\boldsymbol{x} ; t) u(\boldsymbol{x} ; t+\Delta t)}}{\overline{u^{2}(\boldsymbol{x})}},
$$

over the mesh and grit roughness at $(y-d) / h \sim 0.15$, corresponding approximately to the peak in $\overline{u^{2}}$, as well as at $(y-d) / h=0.6$. Smooth boundary-layer data at $R e_{\tau}=7610$ from Hutchins \& Marusic (2007) are also included: they show that the correlations are highly insensitive to $R e_{\tau}$. The flow over the grit surface exhibits a correlation of larger absolute magnitude than that over the mesh for all $y / h \lesssim 0.6$. The streamwise periodicity of the mesh $\left(L_{x} / h \sim 0.25\right)$ does not appear to affect the correlations. While the correlations do not exhibit a roughness-dependence above $y / h=0.6$, the differences between rough channel flow and smooth boundary layers is largest in the outer regions. The correlation lengths at all $y / h$ are longer than those observed in a boundary layer by Hutchins \& Marusic (2007) and the difference increases with wall-normal distance; this can be attributed to the difference in the boundary conditions (Monty et al. 2007). Dennis \& Nickels (2008) have noted that the use of Taylor's hypothesis for the deduction of structural information in smooth-wall flows will be limited for separations larger than about $6 \delta$ : on rough surfaces, this limitation is likely to be even more severe (Birch \& Morrison 2010). 

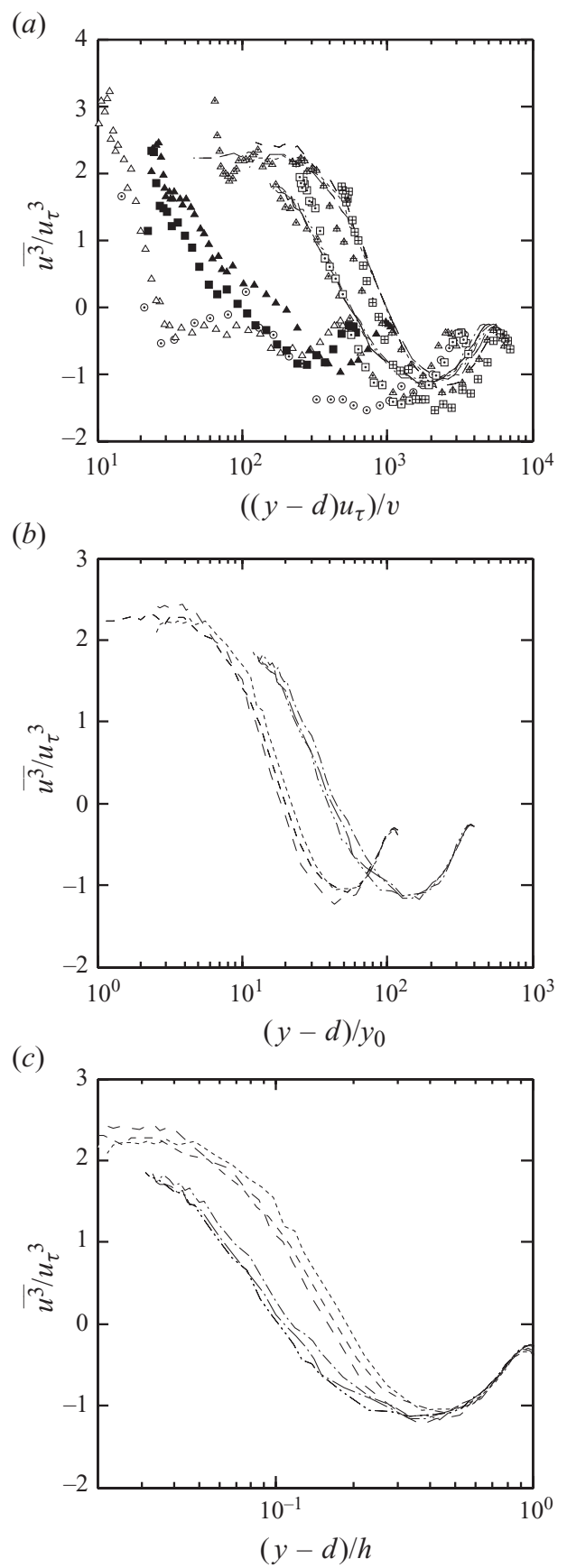

FIGURE 12. Third moment $\overline{u^{3}}:(a)$ viscous scaling, $(b)$ inner scaling and $(c)$ outer scaling. Symbols are data from Bakken et al. (2005).

The two-point spatial correlation for the $u$-component velocity fluctuation given by

$$
R_{11}(y+\Delta y, z+\Delta z)=\frac{\overline{u(y, z) u(y+\Delta y, z+\Delta z)}}{\overline{u(y, z)^{2}}}
$$



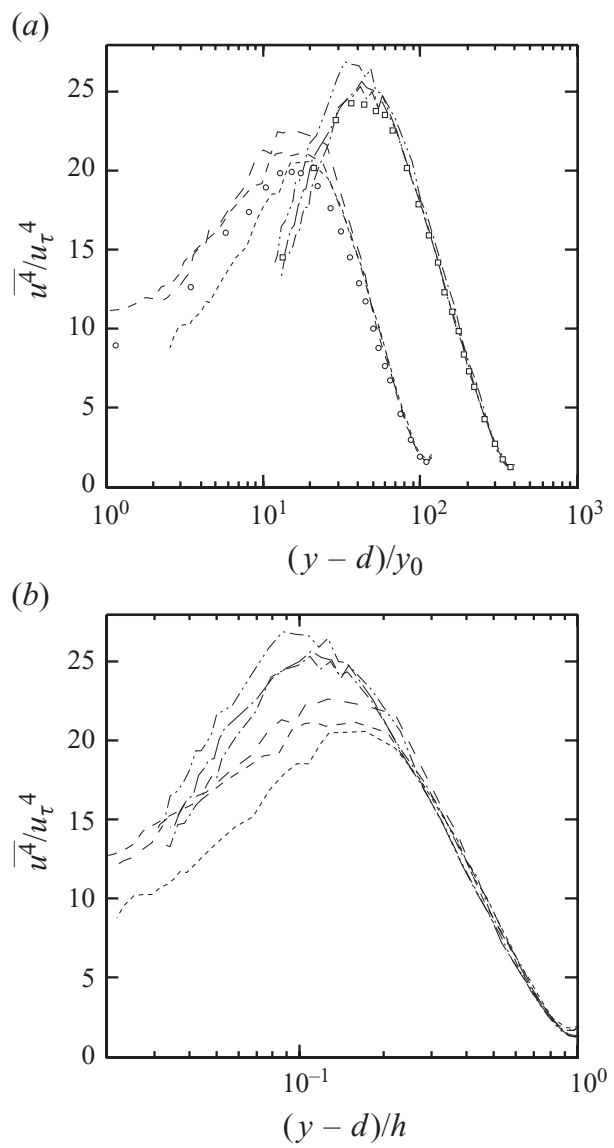

FIGURE 13. Fourth moment $\bar{u}^{+}:(a)$ inner scaling and $(b)$ outer scaling. Symbols are data taken from very long samples $\left(T^{+}=T u_{\tau}^{2} / v=3.0 \times 10^{8}\right.$ and $3.8 \times 10^{8}$ for the grit $(\square)$ and mesh (○) surfaces, respectively, corresponding to $2^{24}$ data points collected at $20 \mathrm{kHz}$ ).

is examined, primarily to investigate differences in large structure between the mesh and grit surfaces. Here, the fixed probe is located at $(y, z)$, on the channel centreline at the wall-normal distance at which $\overline{u^{2}}$ is a maximum $(y / h \approx 0.2)$ with spatial separations $(\Delta y, \Delta z)$ in the crossflow plane (figure 16). Some noise was observed at $\Delta z=0$ for large $\Delta y$, which was the result of interference from the fixed probe support on the moving probe. As with the longitudinal auto-correlations, the twopoint correlations are stronger over the grit surface than over the mesh surface, and distinct differences in the structure of the flow are apparent for $y / h \lesssim 0.6$. Over the mesh surface, Townsend's 'backflow' region is broader and of lower magnitude. It is also apparent that the inclination of the large structure towards the wall is more acute in the case of the mesh; the angle subtended between the axis of structure and the wall decreases from approximately $67^{\circ}$ over the grit to $62^{\circ}$ over the mesh.

Figure 17 shows the two-point spanwise correlation function, $R_{11}(0, \Delta z)$, at $y / h=0.14,0.4$ and 0.8 , calculated with a pair of single hot wires: it compares the results with the hot-wire rake data of Hutchins \& Marusic (2007) in a smooth-wall boundary layer for $1000 \leqslant R e_{\tau} \leqslant 20000$, and those from a smooth pipe by Monty et al. (2007). The present results for each roughness agree well: the correlation is 


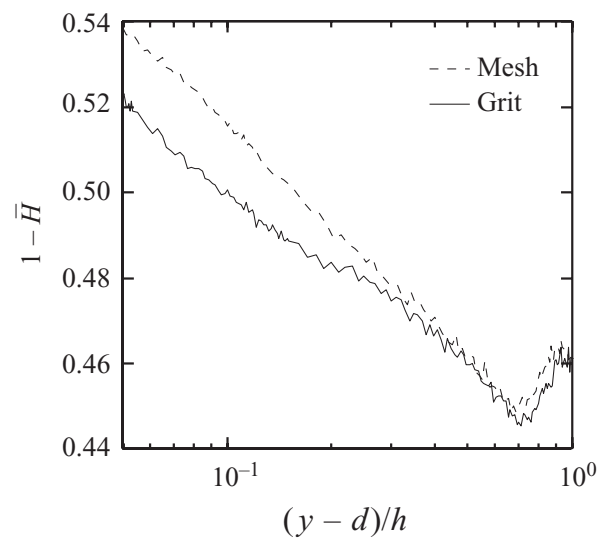

FIGURE 14. Wall-normal variation of the time-mean Heaviside function over the mesh- and grit-type roughnesses $\left(R e_{\tau}=5830\right.$ and 5130 , respectively).

(a)

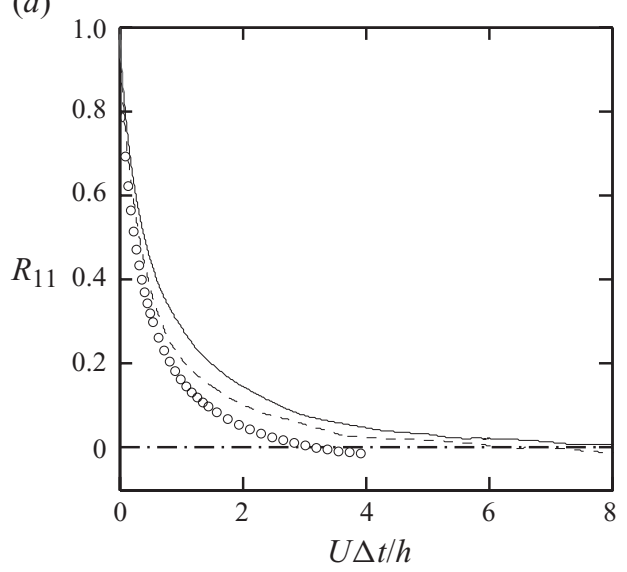

(b)

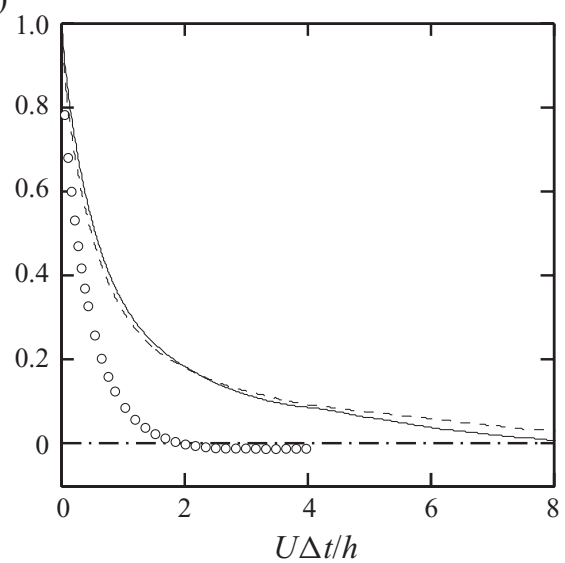

FIGURE 15. Streamwise auto-correlation function evaluated at $(a) y / h \sim 0.15$ and $(b)$ $y / h \sim 0.6$. Lines and symbols: - - grit-type roughness, $R e_{\tau}=5130 ;---$, mesh-type roughness, $R e_{\tau}=5830 ; O$, boundary-layer data from Hutchins \& Marusic (2007) at $\operatorname{Re}_{\tau}=7610(\mathrm{y} / \delta=0.15$ and 0.5 ).

not strongly dependent on the roughness details. The correlation minima of all three studies have a similar magnitude of $R_{11} \approx-0.2$. Monty et al. (2007) define a 'spanwise width scale', $l_{z}$, as the change in value of the abscissa over which $R_{11}( \pm \Delta z) \geqslant 0.05$ : note that this is a measure of the largest eddy contributing to a positive correlation (crudely, the diameter of a large eddy with circulation in the $(y, z)$-plane), while the integral correlation length scale is a measure of the average eddy size contributing to all of the correlation. Estimates of $l_{z}$ for internal flows are larger than those for boundary layers (Monty et al. 2007). The present results for the rough-wall channel show an even broader region of positive $R_{11}$ than for smooth channels: here $l_{z} / h \approx 0.61,0.77$ and 1.0 at $y / h=0.14,0.4$ and 0.8 , respectively, while the data of Hutchins \& Marusic (2007) for a smooth channel are consistently lower. This demonstration of large-scale organization in the presence of a very rough surface has yet to be fully explained. 

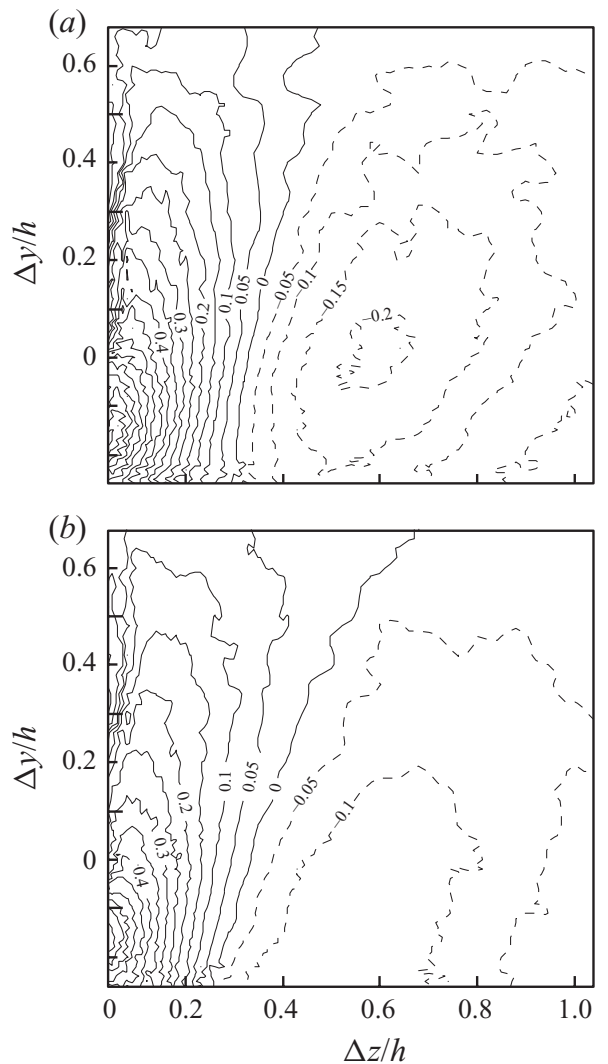

FIGURE 16. Two-point spanwise and wall-normal correlation fields over $(a)$ the mesh and (b) grit roughness; $R e_{\tau}=5830$ and 5130 , respectively.

(a)

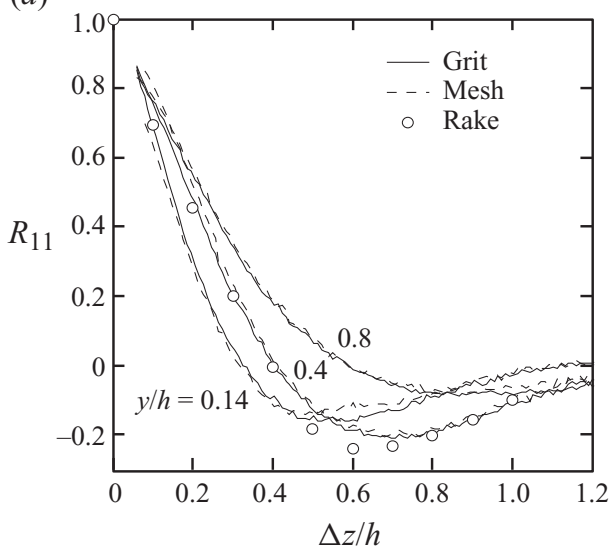

(b)

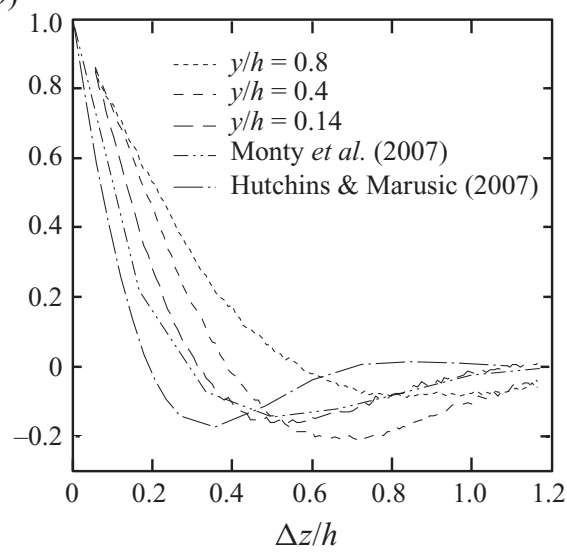

FIGURE 17. Two-point spanwise correlations. (a) Dual single-wire results for cases G2 and M2; hot-wire rake correlations at $y / h=0.4$ for case $\mathrm{G} 2$ are also included for comparison. (b) Comparison of present results (case G2) with the collapsed boundary-layer results of Hutchins \& Marusic (2007) at $y / \delta=0.2$, and the pipe results of Monty et al. (2007) at $y / R=0.15$. 


\section{Discussion and conclusions}

Our most important result is that large roughness with large-scale periodic geometry ('2.5D' roughness) leads to a lack of self-similarity in the mean velocity profile as evidenced by the log law. The lack of simultaneous collapse on the inner $\left(u_{\tau}, y_{0}\right)$ and outer $\left(u_{\tau}, h\right)$ scaling appears to be primarily due to the lack of collapse on outer scales for $(y-d) / h \lesssim 0.30$, which is at distances from the surface that are larger than the maximum distance at which the effects of spanwise inhomogeneity in the mean velocity are apparent, $(y-d) / h \lesssim 0.08$. This result appears not to have been noticed previously and may be attributed in large measure to the fact that measurements close to a very rough surface are inherently difficult to make, not only because of the roughness topology, but also because the Reynolds number is large. There is the additional question of the uncertainty associated with the estimates of the roughness length, $y_{0}$, and offset, $d$. Therefore, in the Appendix, we provide a detailed explanation of how these parameters were estimated and the likely error. A variation in $\kappa=0.41 \pm 0.01$ leads to uncertainty in the values of the roughness length, $y_{0}$, and offset, $d$, which is less than the overall experimental error. It is possible that the apparent lack of self-similarity in the mean velocity profile on the mesh surface could be accommodated by a change in $\kappa$ outside this range. This would then imply that the value of $\kappa$ depends on the type of roughness. It is often the case that self-similarity is assumed once collapse on inner scales alone has been demonstrated. Obviously, in the case of the atmospheric surface layer, demonstration of collapse on outer variables is not possible.

Tani (1987) has suggested that the wake strength is unaffected by the effects of roughness, these being solely represented by the Hama roughness function, $\Delta U^{+}$. The present data show that the wake strength increases slightly on the grit surface, but that it is reduced on the mesh surface. The wake strength, however, may only be defined once the log law is clearly demonstrated. Castro (2007) has shown that in the case of boundary layers, the Coles wake parameter, estimated from an integration of the mean velocity profile, increases with roughness offset. In spite of clear changes in the nature or strength of the wake, Townsend's outer similarity does hold: in the present case, profiles of $\overline{u^{2}}$ collapse for $(y-d) / h \gtrsim 0.2$, but this increases to 0.4 when the data of Bakken et al. (2005) are included.

Streamwise velocity correlations in the crossflow plane show clear evidence of large-scale streamwise structure such as those observed by Ganapathisubramani et al. (2005) and Hutchins \& Marusic (2007) in the log region of boundary layers on smooth walls. The existence of large streamwise structures over fully rough walls has already been noted by Hutchins \& Marusic (2007). Here we provide firm evidence of their existence, although for their eduction, spatial correlations rather than time histories are preferable. Here, we show no evidence of their streamwise extent. However, in Birch \& Morrison (2010), we show evidence of large structures on the grit surface with an approximate streamwise extent of $15 \mathrm{~h}$, deduced from time histories measured by a hot-wire rake. It is likely that their origin is due to the mean strain rate rather than the near-wall cycle characteristic of smooth-wall flows. The clear and distinguishable negative peaks in the two-point longitudinal velocity correlations are consistent with the presence of these structures, and their persistence with circulation in the $(y, z)$-plane has been clearly demonstrated. It seems likely that they are related to the non-normality of the three-dimensional small-perturbation equations (see for example del Álamo \& Jiménez 2006; Cossu, Pujals \& Depardon 2009). 
There is already evidence that the small-scale near-wall structures and these larger structures are able to interact (Toh \& Itano 2005). The dependence of the longitudinal correlations upon the roughness geometry (in wall layers with similar $\Delta U^{+}$) indicates that the roughness can perturb the trajectories of these structures in a manner which is topology-specific. Since evidence of these large structures persists in the velocity statistics to wall-normal distances as large as $y / h($ or $y / \delta) \sim 0.5$, information about the wall geometry may be carried into the core flow without requiring more significant inner-outer interaction. This effect is not apparent in the mean velocity profiles. However, the second-order velocity moment is sensitive to the directionality detected by the Heaviside equation: although the effect is small, the mesh appears to produce $u$-component fluctuations that are more likely to be negative. This seems likely to account for the observation that the inclination of the large structure towards the wall is more acute in the case of the mesh.

The support of the Engineering and Physical Sciences Research Council, UK, under grant number EP/D037166 is gratefully acknowledged. D.M.B. was partially supported by a fellowship from the Natural Sciences and Engineering Research Council of Canada (329117/2006). We are very grateful for highly instructive conversations with I. Castro and K. Flack and to P.-A. Krogstad and J. P. Monty for providing their data.

\section{Appendix. Uncertainty in the log-law constants}

In order to determine a value of the log-law constants as a function of $d$, it was first necessary to detect the region in which the mean flow profile is logarithmic. To accomplish this, the slope of the log-scaled velocity profile was computed as a central difference, or

$$
\frac{\partial U(y)}{\partial \ln (y-d)} \approx \frac{1}{u_{\tau}} \frac{U(y+\Delta y)-U(y-\Delta y)}{\ln (y+\Delta y-d)-\ln (y-\Delta y-d)} .
$$

In the logarithmic region, (1.2) must be satisfied; therefore, in this region,

$$
\frac{\partial U(y)}{\partial \ln (y-d)}=\frac{1}{\kappa} \text {. }
$$

The value of $\kappa$ was taken here as 0.41 . An objective function $f(y-d)$ could then be constructed by combining (A 1) and (A 2), as

$$
f(y-d)=\left(\frac{1}{u_{\tau}} \frac{U(y+\Delta y)-U(y-\Delta y)}{\ln (y+\Delta y-d)-\ln (y-\Delta y-d)}-\frac{1}{k}\right)^{2} .
$$

After applying a simple, rectangular-window filter to minimize the propagation of experimental noise, the logarithmic region was identified as the region in which $f(y-d)<\epsilon$, where $\epsilon$ is some threshold value (as illustrated in figure 18). A very high accuracy in the range of the logarithmic region is not necessarily required, as the $\log$ law is expected to hold true anywhere within the range.

Once a log region had been detected, the log-law offset could be determined from (1.2), as

$$
y_{0}=(y-d) \exp \left(-\kappa \frac{U}{u_{\tau}}\right)
$$

This expression indicates that $y_{0}$ is a function of the wall-normal distance; however, $y_{0}$ is expected to be constant throughout the logarithmic region. A unique value of 


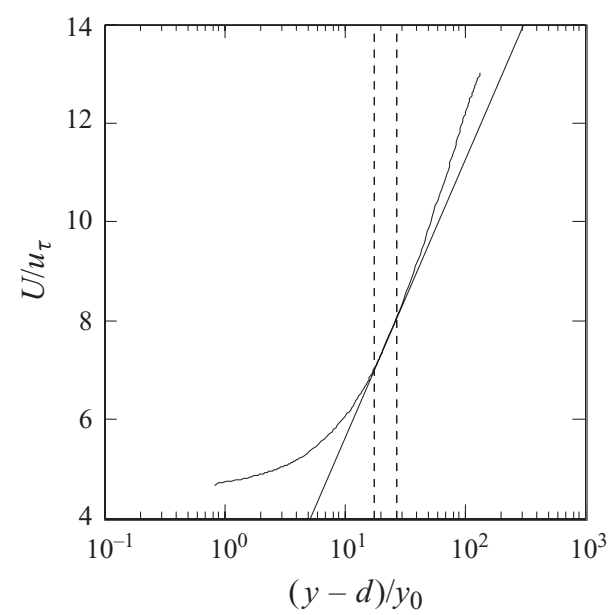

Figure 18. Detection of the logarithmic region in the mean flow over the mesh-type roughness.

(a)

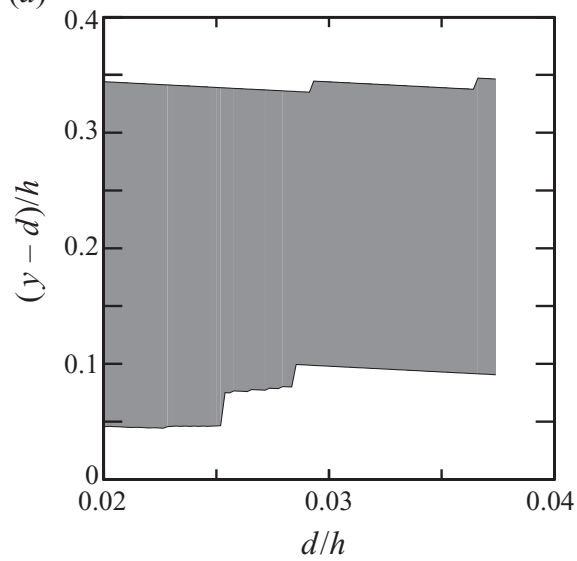

(b)

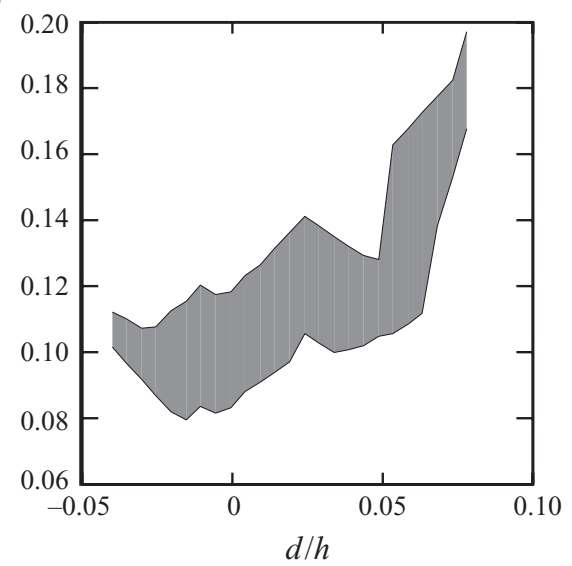

FIGURE 19. Range of the logarithmic region detected over the $(a)$ grit-type and $(b)$ mesh-type roughnesses as a function of $d$.

$y_{0}$ may then be obtained simply as the bounded spatial average,

$$
y_{0}=\frac{1}{y_{2}-y_{1}} \int_{y_{1}}^{y_{2}}(y-d) \exp \left(-\kappa \frac{U(y)}{u_{\tau}}\right) \mathrm{d} y,
$$

where $y_{1}$ and $y_{2}$ are the lower and upper bounds of the logarithmic region, respectively.

The range of the logarithmic region detected as described above was plotted as a function of the origin shift $d$ in figure 19, for the cases of the grit-type roughness (at $\left.R e_{\tau} \sim 5130\right)$ and the mesh-type roughness $\left(R e_{\tau} \sim 5830\right)$.

In general, the flow over the grit-type roughness exhibited a much wider logarithmic region which was fairly insensitive to the value of $d$. On the other hand, the flow over the mesh had a very narrow logarithmic region for all values of $d$, and the wallnormal distance to the logarithmic region increased fairly rapidly with increasing $d$. The very narrow logarithmic region was, in many cases, equivalent to the range 


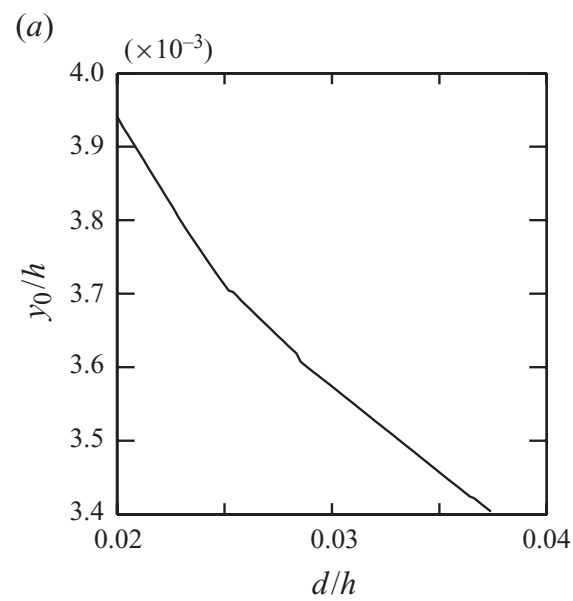

(b)

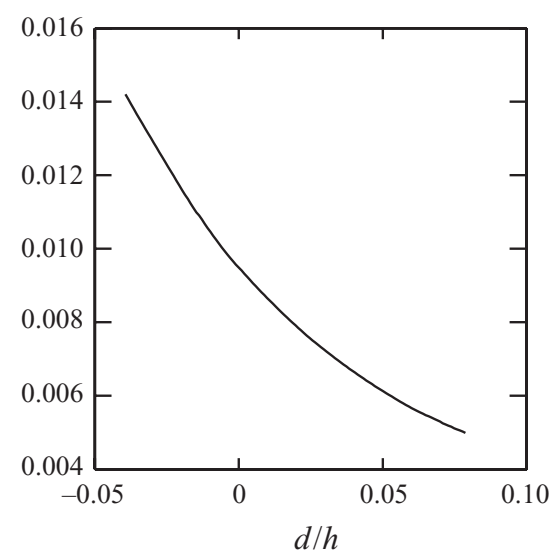

FIGURE 20. Variation of the inner-scaled velocity offset $y_{0}$ with $d$ over the $(a)$ grit-type and (b) mesh-type roughnesses.

of the threshold $\epsilon$, indicating that the detected logarithmic region was, to within experimental uncertainty, a point of tangency only.

It should further be noted that the variation of the bounds of the log region, $y_{1}$ and $y_{2}$, was in both cases monotonic in $d$ (to within the experimental uncertainty); consequently, determining a unique value of $y_{1}$ or $y_{2}$ for a given data set is equivalent to determining a unique value of $d$.

The velocity offset $y_{0}$ was then determined within the logarithmic region detected, and was plotted as a function of $d$ in figure 20 for all values of $d$ in which a $\log$ region existed with a slope of $\kappa$.

The velocity shift was considerably less sensitive to the uncertainty of the origin for the case of the grit-type roughness, exhibiting an approximate slope of $\partial y_{0} / \partial d \sim-0.03$ compared to $\partial y_{0} / \partial d \sim-0.08$ for the mesh-type roughness. These result in ranges of uncertainty in $y_{0}$ of $\sim \pm 7$ and $\sim \pm 50 \%$, respectively.

The variation of the viscous- and outer-scaled log-law offsets, $\Delta U^{+}$and $D$, may be equally determined from these results using (1.3) and (1.5). However, when scaled on outer or viscous parameters, the velocity offset will be much less sensitive to changes in $d$ since both $\Delta U^{+}$and $D$ vary with $\ln \left(y_{0}\right)$.

\section{REFERENCES}

Del Álamo, J. C. \& JimÉnez, J. 2006 Linear energy amplification in turbulent channels. J. Fluid Mech. 559, 205-213.

Antonia, R. A. \& Krogstad, P. Å. 2001 Turbulence structure in boundary layers over different types of surface roughnesses. Fluid Dyn. Res. 28, 139-157.

Bakken, O. M., Krogstad, P. Å., Ashrafian, A. \& Andersson, H. I. 2005 Reynolds number effects in the outer layer of the turbulent flow in a channel with rough walls. Phys. Fluids 17, 065101.

Bhaganagar, K., Kim, J. \& Coleman, G. 2004 Effect of roughness on wall-bounded turbulence. Flow Turbul. Combust. 72, 463-492.

Birch, D. \& Morrison, J. F. 2010 Hot-wire measurements in rough-wall turbulent channel flow using an innovative monolithic CTA. J. Ind. Aero. Wind Engng (submitted).

BradshaW, P. 1967 'Inactive' motion and pressure fluctuations in turbulent boundary layers. J. Fluid Mech. 30, 241-258.

BREUER, K. S. 1995 Stochastic calibration of sensors in turbulent flow fields. Exp. Fluids 19 (2), $138-141$. 
CAstro, I. P. 2007 Rough-wall boundary layers: mean flow universality. J. Fluid Mech 585, 469-485.

Clauser, F. H. 1956 The turbulent boundary layer. Adv. Appl. Mech. 4, 1-51.

Colebrook, C. F. \& White, C. M. 1937 Experiments with fluid friction in roughened pipes. Proc. R. Soc. Lond. A 161, 367-381.

Connelly, J. S., Schultz, M. P. \& Flack, K. A. 2006 Velocity defect scaling for turbulent boundary layers with a range of relative roughness. Exp. Fluids 40, 188-195.

Cossu, C., Pujals, G. \& Depardon, S. 2009 Optimal transient growth and very large-scale structures in turbulent boundary layers. J. Fluid Mech. 619, 79-94.

Dennis, D. J. C. \& Nickels, T. B. 2008 On the limitations of Taylor's hypothesis in constructing long structures in a turbulent boundary layer. J. Fluid Mech. 614, 197-206.

Flack, K. A., Schultz, M. P. \& Connelly, J. S. 2007 Examination of a critical roughness height for outer layer similarity. Phys. Fluids 19, 095104.

Flack, K. A., Schultz, M. P. \& Shapiro, T. A. 2005 Experimental support for Townsend's Reynolds number similarity hypothesis on rough walls. Phys. Fluids 17, 035102.

Ganapathisubramani, B., Hutchins, N., Hambleton, W. T., Longmire, E. K. \& Marusic, I. 2005 Investigation of large-scale coherence in a turbulent boundary layer using two-point correlations. J. Fluid Mech. 524, 57-80.

Hama, F. 1954 Boundary-layer characteristics for smooth and rough surfaces. Trans. Soc. Naval Arch. Marine Engng 62, 333-358.

Hunt, J. C. R. \& Morrison, J. F. 2000 Eddy structure in turbulent boundary layers. Eur. J. Mech. B. Fluids 19, 673-694.

Hutchins, N. \& Marusic, I. 2007 Large-scale influences in near-wall turbulence. Philos. T. R. Soc. A 365 (1852), 647-664.

JACKSON, P. S. 1981 On the displacement height in the logarithmic velocity profile. J. Fluid Mech. 111, 15-25.

JimÉnez, J. 2004 Turbulent flows over rough walls. Annu. Rev. Fluid Mech. 36, 173-196.

Keirsbulck, L., Labraga, L., Mazouz, A. \& Tournier, C. 2002 Surface roughness effects on turbulent boundary layer structures. J. Fluids Engng 124, 127-135.

Krogstad, P. Å., Andersson, H. I., Bakken, O. M. \& Ashrafian, A. 2005 An experimental and numerical study of channel flow with rough walls. J. Fluid Mech. 530, 327-352.

Krogstad, P. Å. \& Antonia, R. A. 1994 Structure of turbulent boundary layers on smooth and rough walls. J. Fluid Mech. 277, 1-21.

Krogstad, P. Å., Antonia, R. A. \& Browne, L. W. B. 1992 Comparison between rough- and smooth-wall turbulent boundary layers. J. Fluid Mech. 245, 599-617.

Leonardi, S. \& Castro, I. P. 2010 Channel flow over large cube roughness: a direct numerical simulation study. J. Fluid Mech. 651, 519-539.

Li, J. D., McKeon, B. J., Jiang, W., Morrison, J. F. \& Smits, A. J. 2004 The response of hot wires in high Reynolds-number turbulent pipe flow. Meas. Sci. Technol. 15, 1-10.

McKeon, B. J., Li, J. D., Jiang, W., Morrison, J. F. \& Smits, A. J. 2004 Further observations on the mean velocity distribution in fully developed turbulent pipe flow. J. Fluid Mech. 501, $135-147$.

McKeon, B. J., Zagarola, M. V. \& Smits, A. J. 2005 A new friction factor relationship for fully developed pipe flow. J. Fluid Mech. 538, 429-443.

Monty, J. P. 2005 Developments in smooth wall turbulent duct flows. PhD thesis, University of Melbourne.

Monty, J. P., Stuart, J. A., Williams, R. C. \& Chong, M. S. 2007 Large-scale features in turbulent pipe and channel flows. J. Fluid Mech. 589, 147-156.

Morrison, J. F. 2007 The interaction between inner and outer regions of turbulent wall-bounded flow. Philos. Trans. R. Soc. A 365 (1852), 683-698.

Morrison, J. F., Subramanian, C. S. \& Bradshaw, P. 1992 Bursts and the law of the wall in turbulent boundary layers. J. Fluid Mech. 241, 75-108.

NikURADSE, J. 1933 Strömungsgesetze in rauhen Rohren. Tech. Rep. 361. Forsch. Arb. Ing.-Wes. (English translation: Laws of flow in rough pipes, NACA TM 1292.)

Raupach, M. R., Antonia, R. A. \& Rajagopalan, S. 1991 Rough-wall turbulent boundary layers. Appl. Mech. Rev. 44 (1), 1-25.

RotTA, J. C. 1962 Turbulent boundary layers in incompressible flow. Prog. Aero. Sci. 2, 1-219. 
Schultz, M. P. \& Flack, K. A. 2007 The rough-wall turbulent boundary layer from the hydraulically smooth to the fully rough regime. J. Fluid Mech 580, 381-405.

Shockling, M. A., Allen, J. J. \& Smits, A. J. 2006 Roughness effects in turbulent pipe flow. J. Fluid Mech. 564, 267-285.

TANI, I. 1987 Turbulent boundary layer development over rough surfaces. In Perspectives in Turbulence Studies (ed. H. U. Meier \& P. Bradshaw), pp. 223-249. Springer.

ToH, S. \& Itano, T. 2005 Interaction between a large-scale structure and near-wall structures in channel flow. J. Fluid Mech. 524, 242-262.

Townsend, A. A. 1956 The Structure of Turbulent Shear Flow, 1st edn. Cambridge University Press.

Townsend, A. A. 1961 Equilibrium layers and wall turbulence. J. Fluid Mech. 11, 97-120.

Townsend, A. A. 1976 The Structure of Turbulent Shear Flow, 2nd edn. Cambridge University Press.

Volino, R. J., Schultz, M. P. \& FlaCK, K. A. 2007 Turbulence structure in rough- and smooth-wall boundary layers. J. Fluid Mech. 592, 263-293.

Wu, Y. \& Christensen, K. T. 2007 Outer-layer similarity in the presence of a practical rough-wall topography. Phys. Fluids 19, 085108.

Zagarola, M. V. \& SMits, A. J. 1998 Mean-flow scaling of turbulent pipe flow. J. Fluid Mech. 373, 33-79. 